\title{
CRISPR-based functional genomics in human dendritic cells
}

$6{ }^{1}$ Department of Cellular and Molecular Pharmacology, University of California, San Francisco,

7 San Francisco, California, USA.

$8{ }^{2}$ Howard Hughes Medical Institute, University of California, San Francisco, San Francisco,

9 California, USA.

$10{ }^{3}$ California Institute for Quantitative Biosciences, University of California, San Francisco, San

11 Francisco, California, USA

$12{ }^{4}$ Department of Microbiology and Immunology, University of California, San Francisco, San

13 Francisco, California, USA.

$14{ }^{5}$ Department of Bioengineering, Stanford University, Stanford, California, USA

$15{ }^{6} \mathrm{ChEM}-\mathrm{H}$, Stanford University, Stanford, California, USA

$16 \quad{ }^{7}$ Chan Zuckerberg Biohub, San Francisco, California, USA

$17{ }^{8}$ Whitehead Institute for Biomedical Research, Cambridge, Massachusetts, USA

$18{ }^{9}$ Department of Biology, Massachusetts Institute of Technology, Cambridge, Massachusetts,

19 USA

$21 \quad$ \# These authors contributed equally

$22 *$ Correspondence should be addressed to: weissman@wi.mit.edu (JSW),

23 fischbach@fischbachgroup.org (MAF) 


\section{Abstract}

25 Dendritic cells (DCs) regulate processes ranging from antitumor and antiviral immunity to host-

26 microbe communication at mucosal surfaces. It remains difficult, however, to genetically

27 manipulate human DCs, limiting our ability to probe how DCs elicit specific immune responses.

28 Here, we develop a CRISPR/Cas9 genome editing method for human monocyte-derived DCs

29 (moDCs) that mediates knockouts with a median efficiency of $>93 \%$ across $>300$ genes. Using

30 this method, we perform genetic screens in moDCs, identifying mechanisms by which DCs tune

31 responses to lipopolysaccharides from the human microbiome. In addition, we reveal donor-

32 specific responses to lipopolysaccharides, underscoring the importance of assessing immune

33 phenotypes in donor-derived cells, and identify genes that control this specificity, highlighting

34 the potential of our method to pinpoint determinants of inter-individual variation in immune

35 responses. Our work sets the stage for a systematic dissection of the immune signaling at the

36 host-microbiome interface and for targeted engineering of DCs for neoantigen vaccination. 


\section{Introduction}

39 DCs play an outsized role in orchestrating innate and adaptive immunity: they act as sentinels,

40 detecting invaders and initiating innate immune responses to clear them, and as antigen-

41 presenting cells, initiating adaptive immune responses that are antigen-specific and tailored to

42 the context in which the antigen was detected (Merad et al., 2013; Sun et al., 2020). In this

43 fashion, DCs mediate pathogen clearance, tumor cell killing, and tolerance to microbiome

44 bacteria or dietary antigens. DCs thus play fundamental roles in shaping host-pathogen and host-

45 microbiome interactions and in the etiology of autoimmune disorders and are a major target for

46 efforts to develop new generations of immunotherapies (Wculek et al., 2020).

47 Dissecting the pathways by which human DCs respond to innate immune stimuli and

48 relay them into adaptive responses, however, has been challenging, due in large part to

49 difficulties in genetically manipulating human DCs. Although approaches for gene repression in

50 human DCs by RNAi have been reported (Song, 2014), RNAi suffers from limited efficacy and

51 specificity, precluding broader implementation (Kaelin, 2012). As a consequence, DC biology is

52 generally studied in mouse models, but mice and humans differ in many aspects of both innate

53 and adaptive immunity, including innate immune receptor repertoires, responses to immune

54 ligands such as lipopolysaccharide (LPS), and developmental pathways of adaptive immune cells

55 (Pulendran and Davis, 2020). One way to address this challenge is to knock out genes in DC

56 precursor populations such as monocytes or stem cells, followed by differentiation into DCs

57 (Freund et al., 2020; Hiatt et al., 2020; Laustsen et al., 2018). These methods, however, require

58 independent differentiation of each knockout population and as a result are susceptible to batch

59 effects and poorly suited for genetic screens. Moreover, they do not permit probing the functions

60 of genes required for DC differentiation and culture. More broadly, both animal and stem cell 
61 models fail to capture inter-individual variation in immune phenotypes (Sanz et al., 2018), which

62 has been observed for example in innate immune responses, autoimmunity, and pathogen

63 susceptibility and has gained particular salience during the Covid-19 pandemic (Lucas et al.,

64 2020; Pereira et al., 2020). Such variation results from a combination of genetic factors and

65 lifelong environmental exposures (e.g., from the microbiome) but it remains challenging to

66 define the causal determinants in the absence of genetic tools for patient-derived immune cells

67 such as DCs.

To address these limitations, we developed a CRISPR/Cas9-based strategy to construct

69 targeted knockouts directly in human monocyte-derived DCs (moDCs), which are readily

70 derived from donor blood and are widely used for research and clinical applications (Sallusto and

71 Lanzavecchia, 1994; Garg et al., 2017). Using this strategy, we conducted a genetic screen for

72 factors that recognize an innate immune ligand from the human microbiome, LPS from the gut

73 commensal Bacteroides thetaiotaomicron (B. theta), and identified both mechanisms of species-

74 specific LPS recognition and factors that give rise to inter-individual variation in the response to

75 LPS. These results highlight the utility of our strategy in identifying receptors for innate immune

76 ligands, such as those from the human microbiome, and in pinpointing the genetic bases of inter-

77 individual variation in human immunity. More broadly, our work now provides a general

78 blueprint for functional genomics in human DCs and enables targeted DC engineering for

79 deployment in immunotherapy. 


\section{Results}

85 A CRISPR/Cas9 strategy for functional genomics in moDCs

86 To enable introduction of specific knockouts in moDCs, we developed a non-viral genome

87 editing strategy based on electroporation of in vitro-assembled Cas9-sgRNA complexes (Cas9

88 ribonucleoprotein particles, RNPs), an approach that has been validated in other immune cell

89 types (Freund et al., 2020; Hiatt et al., 2020; Riggan et al., 2020; Roth et al., 2018; Schumann et

90 al., 2015). Briefly, our strategy entails isolating monocytes from human donor blood,

91 differentiating them into moDCs in the presence of GM-CSF and IL-4, and electroporating these

92 moDCs with Cas9 RNPs to induce double-strand breaks at the targeted locus (Figure 1a). Such

93 double-strand breaks trigger error-prone DNA repair and subsequent formation of insertions or

94 deletions (indels) that, with a certain frequency, cause frameshift mutations and thus knockout of

95 the targeted gene. We monitor the efficiency of this process by genotyping using next-generation

96 sequencing and by phenotyping using functional assays.

97 We first electroporated moDCs with Cas9 RNPs targeting AAVS1, using a validated

98 sgRNA sequence (Mali et al., 2013), as well as TNF and TLR4 with sgRNA sequences from the

99 Brunello library (Supplementary File 1) (Doench et al., 2016). By testing a grid of

100 electroporation conditions, we identified conditions with efficient genome editing of $A A V S 1$ and

$101 T N F$ and limited toxicity, but editing of TLR4 was inefficient (Figure 1b-c, Figure 1 - Figure

102 Supplement 1a-b, Supplementary File 2). The large majority of observed indels were 1- or 2-bp

103 deletions (Figure 1d, Figure 1 - Figure Supplement 1c), which are frameshift mutations that

104 eliminate the function of the gene.

105 To improve editing efficiency, we i) leveraged sgRNA design tools optimized for RNP

106 activity (CRISPR Design Tool, Synthego) and ii) targeted each locus with 2-3 sgRNAs with 
107 binding sites tiled across a 200-bp stretch to induce simultaneous double-strand breaks, a design

108 that increases the likelihood of achieving functional knockouts by preventing error-free DNA

109 repair and/or removing a stretch of the gene (Methods) (Riggan et al., 2020). Because such large

110 deletions generate smaller amplicons in our genotyping approach, which may be overrepresented

111 in sequencing counts due to length biases in PCR amplification and the sequencing reaction

112 itself, we devised a scheme to correct sequencing counts for length differences to the WT locus

113 to accurately quantify editing efficiency (Figure 1 - Figure Supplement 2, Methods). Testing the

114 multi-sgRNA approach across three loci revealed a shift in indel profiles toward large deletions

115 and multiple indels with deletions of sequences between sgRNA cut sites as well as a modest

116 increase in editing efficiency (Figure 1e, Figure 1 - Figure Supplement 1d-e). Cas9 RNPs

117 assembled with sgRNAs rather than with crRNA:tracrRNA duplexes mediated higher editing

118 efficiency (Figure 1 - Figure Supplement 1d). Through an expansive grid search of

119 electroporation conditions, we next identified a condition (P3, DJ108) with editing efficiencies

$120>90 \%$, high cell recovery, and high specificity in detecting loss of TNF- $\alpha$ secretion upon

121 stimulation with LPS from E. coli O55 (E. coli LPS) after knockout of TNF or the LPS receptor

122 TLR4 (Poltorak et al., 1998) but not CXCR4, a chemokine receptor not involved in LPS signaling

123 (Figure 1 - Figure Supplement 3a). We used this condition for all further experiments, although

124 other conditions also permitted efficient genome editing (Figure 1 - Figure Supplement 3a).

125 Benchmarking the procedure for 10 genes in moDCs derived from three independent donors

126 revealed editing efficiencies $>80 \%$ for all genes and $>90 \%$ for most genes (Figure 1f, Figure 1 -

127 Figure Supplement 3b).

128 In parallel, we challenged knockout moDCs from two donors with E. coli LPS, a TLR4

129 agonist, and measured production of two cytokines: i) TNF- $\alpha$, which is induced by MYD88 and 
130 TRIF (TICAM1) downstream of TLR4, and ii) CXCL10 (IP-10), which is induced solely by

131 TRIF via activation of IRF3 and production of interferon beta (IFNB1) (Fitzgerald et al., 2003;

132 Fitzgerald and Kagan, 2020; Yamamoto et al., 2003, 2002). We normalized cytokine production

133 for each knockout population to that from moDCs with knockout of RPE65, a retinal pigment

134 epithelium-specific gene that does not contribute to DC function and serves as a neutral control.

135 TLR4 knockout abolished production of both TNF- $\alpha$ and CXCL10, knockout of MYD88 or TNF

136 reduced TNF- $\alpha$ production (and mildly increased CXCL10 production in at least one donor),

137 TICAM1 knockout strongly reduced CXCL10 production and moderately reduced TNF- $\alpha$

138 production, and knockout of IFNBI or IRF3 only reduced CXCL10 production (Figure 1, Figure

1391 - Figure Supplement 3c). The effect of knocking out IRF3 was weak, perhaps due to

140 redundancy with other transcription factors such as $I R F 7$ or due to incomplete protein depletion.

141 Separately, knockout moDCs from an independent donor challenged with E. coli LPS or

142 Pam3CSK4, a TLR2/TLR1 agonist, responded as expected: knockout of TNF or MYD88 reduced

143 the response to both stimuli, whereas knockout of TLR4 or TLR2 only reduced the responses to

144 their cognate ligands (Figure 1 - Figure Supplement 3b). Thus, our moDC genome editing

145 strategy enables the detection of functional consequences of knockouts, demonstrating that we

146 can effect protein depletion without perturbing the ability of moDCs to respond to innate

147 immune stimuli.

149 DC responses to LPSs are specific to bacterial species and vary across indviduals

150 We next sought to apply our genome editing strategy to answer a central question in innate

151 immunity. Despite LPS being a classic inflammatory molecule, humans are colonized by trillions

152 of Gram-negative microbes that generate milligram to gram quantities of LPS in their intestinal 
153 tracts without tonic inflammation. This observation has been attributed in part to the different

154 chemical structures and immunomodulatory activities of LPSs from commensal Bacteroidetes,

155 among the most common Gram-negative phyla in the guts of Western individuals (Wexler and

156 Goodman, 2017), compared to the canonical inflammatory LPSs from E. coli and related

157 Proteobacteria (Coats et al., 2011; Tan et al., 2015; Vatanen et al., 2016; d'Hennezel et al.,

158 2017). Indeed, the Bacteroidetes-to-Proteobacteria LPS ratio in the gut microbiome has been

159 associated with the incidence of type 1 diabetes (Vatanen et al., 2016), suggesting that LPSs

160 from the human microbiome contribute to shaping immune function. The biological activities of

161 LPSs from gut Bacteroidetes, however, have remained controversial as they have been reported

162 to be both TLR4 antagonists and agonists (d'Hennezel et al., 2017; Steimle et al., 2019; Vatanen

163 et al., 2016). We set out to establish how human DCs respond to LPS from Bacteroidetes and

164 more broadly how DCs discriminate different LPSs and initiate specific immune responses.

165 We focused on LPS from $B$. theta, an abundant member of the human gut microbiota

166 whose LPS biosynthetic machinery has been characterized, allowing us to genetically manipulate

167 its structure (Coats et al., 2011; Cullen et al., 2015; Jacobson et al., 2018). (B. theta LPS formally

168 is a lipoooligosaccharide, but we refer to it as LPS for clarity.) We purified LPS from a $B$. theta

169 strain carrying deletions of all eight capsular polysaccharide biosynthetic gene clusters (Porter et

170 al., 2017) to obtain LPS without other contaminating glycolipids. Human moDCs stimulated

171 with $B$. theta LPS secreted moderate levels of TNF- $\alpha$ as quantified by ELISA; this response was

172 weaker than that elicited by $E$. coli LPS both in magnitude and apparent EC 50 but substantially

173 stronger than that elicited by Rhodobacter sphaeroides LPS, a well-characterized TLR4

174 antagonist (Figure 2a) (Golenbock et al., 1991). Although the pattern was consistent across

175 moDCs from independent donors, response magnitude and EC50 varied by 6-fold and $>20$-fold, 
176 respectively (Figure 2 - Figure Supplement 1a), even for moDCs processed in parallel,

177 suggesting that donor-specific factors shape immune responses. Analysis of the transcriptional

178 responses of moDCs by RT-qPCR and RNA-seq confirmed that B. theta LPS activated both

179 MYD88 and TRIF signaling more weakly than E. coli LPS, with a more pronounced difference

180 for TRIF signaling, again with donor-to-donor variation (Figure 2b, Figure 2 - Figure

181 Supplement $1 b-c)$.

182

To further establish if $B$. theta LPS is a mild agonist of TLR4 rather than an antagonist,

183 we turned to genetic engineering of $B$. theta LPS. LPSs of Bacteroidetes generally contain

184 pentaacylated, monophosphorylated lipid A as opposed to the hexaacylated, diphosphorylated

185 lipid A of Proteobacteria (Coats et al., 2011; Weintraub et al., 1989), in addition to other

186 differences including lipid A acyl group structures and LPS glycan composition. A previous

187 study had established that $B$. theta LPS modified to contain pentaacylated, diphosphorylated lipid

188 A has increased capacity to stimulate TLR4 signaling via the endocytotic pathway (Tan et al.,

189 2015). Hypothesizing that $B$. theta lipid A further lacking an acyl group would resemble known

190 TLR4 antagonists (Golenbock et al., 1991) and thus have decreased immunostimulatory activity,

191 we stimulated moDCs with LPS purified from a $B$. theta strain genetically engineered to produce

192 tetraacylated, diphosphorylated lipid A (B. theta 4PP LPS) (Jacobson et al., 2018). B. theta 4PP

193 LPS elicited substantially lower levels of TNF- $\alpha$ production and smaller transcriptional

194 responses than $B$. theta WT LPS, with barely detectable responses in some donors (Figure 2a-b,

195 Figure 2 - Figure Supplement 1c). Thus, $B$. theta LPS is a mild TLR4 agonist whose

196 immunostimulatory activity can be tuned by rational engineering. 
200 To determine if the difference in activity between $B$. theta LPS and E. coli LPS arises solely

201 from different capacity to activate TLR4, we tested how TLR4 knockout affects responses to

202 these two LPSs. TLR4 knockout moDCs did not secrete detectable amounts of TNF- $\alpha$ in

203 response to $E$. coli LPS but secreted substantial amounts of TNF- $\alpha$ in response to $B$. theta LPS,

204 corresponding to $30-50 \%$ of the levels secreted by moDCs with knockout of the neutral control

205 gene RPE65 (Figure 2 - Figure Supplement 2a-c). Similarly, TLR4 knockout moDCs retained a

206 partial transcriptional response to $B$. theta LPS corresponding to activation of MYD88, but not

207 TRIF (Figure 2 - Figure Supplement 2d-f). These results suggested that receptors other than

208 TLR4 contribute to recognition of $B$. theta LPS.

To identify such additional receptors, we leveraged our moDC editing strategy to conduct

210 an arrayed genetic screen (Figure 3a). We designed a focused library targeting $\sim 300$ genes

211 including known and predicted pattern recognition receptors and multiple nodes of signaling

212 pathways downstream of each receptor class (Methods). We targeted each gene with 2-3

213 sgRNAs whenever multiple unique sgRNAs could be designed, distributed over four 96-well

214 plates, each of which also included four types of controls (Figure 3 - Figure Supplement 1,

215 Methods). After electroporating moDCs from two independent donors with this library, we

216 assayed editing efficiency and TNF- $\alpha$ secretion in response to $100 \mathrm{ng} / \mathrm{mL} B$. theta LPS

217 (Methods, Supplementary Files 3, 4). Editing efficiency was high in both donors, with median

218 efficiencies of $93 \%$ and $97.6 \%$ (Figure 3b, Figure 3 - Figure Supplement 2-4). TNF- $\alpha$ secretion

219 in response to $B$. theta LPS was strongly reduced by knockouts in the TLR4 signaling pathway,

220 including TLR4 and its co-receptor MD2 (LY96), CD14 (which delivers LPS to TLR4 and

221 initiates TLR4 endocytosis (Zanoni et al., 2011)), as well as genes involved in MYD88 signaling, 


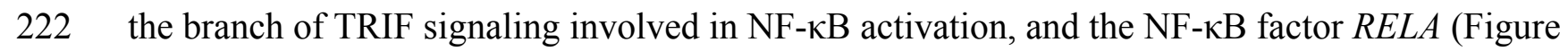

223 3c, Figure 3 - Figure Supplement 5a-c). Indeed, 8-9 of the 10 targeted genes in MYD88

224 signaling reduced TNF- $\alpha$ secretion for both donors. Other knockouts also caused expected

225 phenotypes; for example, moDCs with knockout of A20 (TNFAIP3), which inhibits LPS- and

226 TNF- $\alpha$-induced NF- $\kappa$ B signaling, secreted more TNF- $\alpha$ (Figure 3c, Figure 3 - Figure

227 Supplement 5c). Thus, our genetic screen accurately captured the genetic requirements for the 228 response to $B$. theta LPS.

Although the results for the two donors were similar overall, we noticed several key

230 differences. Most prominently, knockouts of PTPN6 (SHP-1) and to a lesser extent IL10

231 increased TNF- $\alpha$ secretion in response to $B$. theta LPS in one donor (“donor i”) but not the other

232 (“donor h"), suggesting that these factors can constitutively suppress TNF- $\alpha$ secretion in a

233 manner that differs among individuals (Figure 3d). Indeed, unedited moDCs from donor i

234 secreted less TNF- $\alpha$ in response to both $B$. theta LPS and E. coli LPS than those from donor $\mathrm{h}$

235 (Figure 3e). Second, MYD88 signaling contributed particularly strongly to the response to $B$.

236 theta LPS for donor h: knockout of TIRAP, the TLR4-proximal adapter for MYD88 signaling

237 (Fitzgerald et al., 2001; Fitzgerald and Kagan, 2020), induced the strongest decrease in TNF- $\alpha$

238 secretion other than TNF itself. For donor i, TLR4 and the TRIF pathway contributed more

239 strongly to the response, as evidenced by strong decreases in TNF- $\alpha$ secretion upon knockout of

240 TLR4 alone, TRAM (TICAM2), and TRIF (TICAM1) (Figure 3c-d). A separate 40-gene

241 validation experiment with cells from two additional, independent donors recapitulated these

242 results, with results from each of the two validation donors aligning more closely with those

243 from one of the initial donors (Figure 3 - Figure Supplement 6). 
We further investigated two specific observations. First, because TIRAP knockout caused

245 a larger decrease in TNF- $\alpha$ secretion than TLR4 knockout in donor $\mathrm{h}$, we wondered if other TLRs

246 contributed to the response to $B$. theta LPS. We focused on TLR2, canonically known as a

247 receptor for lipopeptides and teichoic acids, because TLR2 knockout caused the next-strongest

248 decrease in TNF- $\alpha$ secretion among TLRs and because TLR2 has been implicated in the

249 response to non-proteobacterial LPSs (Di Lorenzo et al., 2020; Werts et al., 2001), although

250 these claims remain controversial. Indeed, moDCs with simultaneous knockout of TLR4 and

251 TLR2 exhibited the strongest decreases in TNF- $\alpha$ secretion among all samples for both donors

252 (Figure 3f, Figure 3 - Figure Supplement 4d). In addition, knockout of TLR2 alone reduced the

253 response to $B$. theta LPS but not to $E$. coli LPS in a donor in our validation experiment (Figure 3

254 - Figure Supplement 6b). These results are consistent with the possibility that TLR2 contributes

255 to the response to $B$. theta LPS, although we cannot rule out the presence of contaminating

256 lipopeptides in our LPS preparation.

257 Second, to analyze the interplay between PTPN6 and IL-10 in suppressing TNF- $\alpha$

258 secretion in moDCs from donor i, we measured IL-10 levels in the same samples. The effects of

259 knockouts on TNF- $\alpha$ and IL-10 secretion were well-correlated, suggesting that TLR4 signaling

260 via the TRIF and MYD88 branches accounts for secretion of both TNF- $\alpha$ and IL-10. PTPN6

261 stood out as an exception: whereas PTPN6 knockout strongly increased TNF- $\alpha$ secretion, it

262 moderately decreased IL-10 secretion (Figure 3g), suggesting either that IL-10 acts upstream of

263 PTPN6 in suppressing TNF- $\alpha$ secretion or that PTPN6 specifically inhibits production of TNF- $\alpha$

264 and not IL-10. More broadly, these results demonstrate how combining our moDC editing

265 strategy with multiple readouts can increase the resolution in evaluating immune response

266 pathways. 
268 Discussion

269 In summary, we describe an efficient and flexible strategy to introduce knockouts in DCs derived

270 from human donors. Our strategy is compatible with diverse readouts, such as cytokine profiling,

271 RNA-seq, flow cytometry, and microscopy, and enables genetic screens at previously intractable

272 scales, transforming our ability to probe the roles of DCs in human biology. Using our strategy,

273 we derive three main conclusions regarding the recognition of LPSs from the human

274 microbiome. First, we observe a contribution of TLR2 to signaling by $B$. theta LPS, which could

275 result from either an intrinsic ability of $B$. theta LPS to bind both TLR4 and TLR2 or the

276 presence of contaminating TLR2 ligands in our B. theta LPS preparation. Although we cannot

277 rule out contamination, our observation is consistent with a recent report suggesting that $B$.

278 vulgatus LPS binds to TLR2 (Di Lorenzo et al., 2020). Second, moDCs from different donors

279 have distinct responses — both in magnitude and apparent affinity — to identical preparations of

280 LPS, pointing to inter-individual variation in immune responses. Such variation likely results

281 from a combination of genetic factors and environmental exposures that together determine

282 immune cell state, underscoring the importance of probing immune responses directly in donor-

283 derived cells. Third, in PTPN6/SHP-1 we identify a specific factor that contributes to this

284 variation, revealing how our approach enables dissecting the genetic bases of such inter-

285 individual variation. Activation of PTPN6/SHP-1, in addition to regulating innate immunity,

286 suppresses antigen cross-presentation (Ding et al., 2016), which some pathogens exploit to

287 subvert adaptive immunity (C. Khouili et al., 2020). Our finding of inter-individual variation in

288 PTPN6/SHP-1 activity thus has immediate implications both for understanding variations in 
pathogen susceptibility and for personalizing DC vaccines aimed at initiating CD8 T-cell

290 responses.

291 More broadly, we find that human DCs initiate specific responses to LPS from

292 commensal Bacteroidetes, challenging the notion that these LPSs are innocuous components of

293 the human gut microbiota, as further evidenced by a recent report that homeostatic, TLR4-

294 dependent induction of IFN- $\beta$ by $B$. fragilis LPS contributes to antiviral immunity (Stefan et al.,

295 2020). Variations in LPS structure across gut commensals instead alter capacity to activate TLR4

296 and may allow for engagement of new receptors altogether, with the potential for neomorphic

297 activities as well as further complexity arising from combinatorial perception (Antebi et al.,

298 2017). In this fashion, commensal LPSs likely contribute to shaping immune responses at the

299 host-microbiome interface. Understanding the underlying mechanisms, using for example the

300 genetic approaches we describe, may in turn enable efforts to engineer LPSs with defined

301 immunomodulatory capacities, akin to our B. theta 4PP mutant.

302 Beyond LPS recognition, the availability of genetic tools for human DCs now opens the

303 door to a range of applications including systematic functional genomics studies to dissect the

304 roles of DC receptors and signaling pathways in mounting immune responses to commensals,

305 pathogens, or tumor cells and targeted engineering of moDCs for therapeutic interventions such

306 as neoantigen vaccination.

307

308

309 


\section{Materials and Methods}

311 Reagents

312 Complete RPMI medium was generated by supplementing RPMI 1640 medium containing 25

313 mM HEPES, 2 mM L-glutamine, 2 g/L NaHCO 3 (Gibco, Dublin, Ireland) with 10\% (v/v)

314 standard fetal bovine serum (VWR, Wayne, PA), 100 units $/ \mathrm{mL}$ penicillin, $100 \mu \mathrm{g} / \mathrm{mL}$

315 streptomycin, and $2 \mathrm{mM}$ L-glutamine (Gibco). Lyophilized recombinant human GM-CSF

316 (Gemini Bio, Sacramento, CA) and recombinant human IL-4 (Gemini Bio) were reconstituted to

$317100 \mu \mathrm{g} / \mathrm{mL}$ and $40 \mu \mathrm{g} / \mathrm{mL}$, respectively, in sterile $\mathrm{ddH}_{2} \mathrm{O}$, aliquoted into $40-100 \mu \mathrm{L}$ aliquots, and

318 frozen at $-30{ }^{\circ} \mathrm{C}$ until use. Fluorescently labeled antibodies against human CD14 (clone HCD14,

319 PE-, PerCP-Cy5.5-, or BV421-labeled), CD80 (clone 2D10, APC-labeled), CD83 (clone HB15e,

320 APC-Cy7-labeled), CD86 (clone BU63, FITC- or BV605-labeled), HLA-DR (clone L243, PE-

321 or FITC-labeled), CD11b (clone LM2, PE-Cy7-labeled), CD11c (clone Bu15, FITC- or PerCP-

322 Cy5.5-labeled), and CD205/DEC205 (clone HD30, PE-labeled) were obtained from BioLegend

323 (San Diego, CA). Ultrapure LPS from E. coli O55:B5 and Rhodobacter sphaeroides, along with

324 Pam3CSK4, were obtained from Invivogen (San Diego, CA). Solid medium used for bacterial

325 growth was BHI/blood agar, made from Brain Heart Infusion Agar (BD Biosciences, San Jose,

326 CA) with 10\% defibrinated horse blood (Hemostat Laboratories, Dixon, CA). Liquid medium

327 used for bacterial growth was supplemented BHI broth, made by preparing 1 L Brain Heart

328 Infusion Broth (BD), and immediately before starting cultures adding $1 \mathrm{~mL}$ bovine hemin stock

329 (Sigma, St. Louis, MO), $5 \mathrm{mg} / \mathrm{mL}$ in $1 \mathrm{~N}$ sodium hydroxide and filter sterilized, and $10 \mathrm{~mL}$ L-

330 cysteine hydrochloride (Sigma), $50 \mathrm{mg} / \mathrm{mL}$ in Milli-Q water and filter sterilized. Sources of

331 sgRNAs and Cas9 are listed below. 


\section{Bacterial culture}

334 B. theta strains were stored at $-80{ }^{\circ} \mathrm{C}$ in growth medium mixed in equal volume with $50 \%$

335 glycerol in water. Strains were streaked from glycerol stocks onto BHI/blood agar using plastic

336 inoculating loops. Strains were allowed to grow $24-48 \mathrm{~h}$ in an anaerobic chamber. Single

337 colonies were used to inoculate 4 10-mL aliquots of supplemented BHI broth per strain, and after

$33824 \mathrm{~h}$ the $10-\mathrm{mL}$ cultures were expanded to $1 \mathrm{~L}$ each in glass bottles, producing $4 \mathrm{~L}$ total culture

339 volume per strain. Cultures were allowed to grow to stationary phase (24-36 h) and were pelleted

340 at $3400 \times g$ for $1 \mathrm{~h}$ at $4{ }^{\circ} \mathrm{C}$. Pellets were washed in PBS and shipped frozen to the UCSD

341 GlycoAnalytics Core for LPS purification.

\section{B. theta strains}

344 Both the acapsular $B$. theta strain ( $\triangle \mathrm{CPS}$ ) and the $B$. theta 4PP strain have been previously

345 reported (Jacobson et al., 2018; Porter et al., 2017). Briefly, the mutants were created using

346 homologous recombination, creating scarless knockouts of the target genes/gene clusters with no

347 remaining antibiotic resistance markers. The acapsular strain has had all known $B$. theta capsular

348 polysaccharide gene clusters deleted ( 8 clusters in total), and the 4PP strain has had lipid A

349 acyltransferase BT2152 and lipid A phosphatase BT1854 deleted in the acapsular background.

351 LPS purification

352 B. theta LPS preparations were performed by Biswa P. Choudhury at the UCSD GlycoAnalytics

353 Core. A cell pellet from 4 L confluent culture of each B. theta strain was suspended in Milli-Q

354 water and mixed with an equal volume of 90\% phenol solution (Sigma, 328111). The suspension

355 was stirred continuously and maintained at $68^{\circ} \mathrm{C} \pm 2{ }^{\circ} \mathrm{C}$ for $30 \mathrm{~min}$. After cooling in an ice bath, 
356 suspensions were centrifuged at $3500 \mathrm{rpm}$ at $10^{\circ} \mathrm{C}$ for $45 \mathrm{~min}$ and the upper layer removed to a

357 clean Falcon tube. The remaining layers were extracted again with an equal volume of water for

$35830 \mathrm{~min}$, cooled, and centrifuged as before. The upper layers were pooled and dialyzed (1000

359 MWCO, regenerated cellulose tubing) against $4 \mathrm{~L}$ of water for $4 \mathrm{~d}$, replacing the water twice per

360 day. The dialysate was lyophilized, resuspended in water, and subjected to ultracentrifugation at

$361105,000 \times g$ for $4 \mathrm{~h}$. The pellet was resuspended in water, treated with DNase I, RNase A, and

362 proteinase $\mathrm{K}$, followed by another round of ultracentrifugation as above. The resulting pellet was

363 resuspended in water and lyophilized.

366 Human moDCs were differentiated from monocytes isolated from commercially sourced fresh

367 peripheral blood mononuclear cells (PBMCs) from de-identified healthy human donors

368 (AllCells, Alameda, CA). The authors did not obtain identifiable private information on donors.

369 The commercial vendor obtained informed consent from all donors covering all experiments and

370 data reported in this manuscript. Monocytes were isolated from PBMCs by negative magnetic

371 selection using the EasySep human monocyte enrichment kit without CD16 depletion (StemCell)

372 following the manufacturer's instructions and using a Big Easy magnet or Easy 50 magnet

373 (StemCell Technologies, Vancouver, Canada). Enriched monocytes were generally $>80 \%$ CD14-

374 positive, as assessed by flow cytometry on an LSR-II flow cytometer (BD BioSciences) or an

375 Attune NxT flow cytometer (Thermo Fisher Scientific, Waltham, MA). Cell counts were

376 determined in duplicate using a Countess II automated hemocytometer (Thermo Fisher

377 Scientific). The isolated monocytes were cultured in complete RPMI medium, supplemented

378 with $50 \mathrm{ng} / \mathrm{mL}$ GM-CSF and $20 \mathrm{ng} / \mathrm{mL}$ IL-4 immediately prior to use, at a density of $1 \cdot 10^{6}$ to 
$1.3 \cdot 10^{6}$ per $\mathrm{mL}$ at $37^{\circ} \mathrm{C}$ and $5 \% \mathrm{CO}_{2}$ for $7 \mathrm{~d}$. Medium was exchanged every two or three days during this period (twice total). DCs on day 7 were generally positive for CD80, CD83, HLADR, CD11b, CD11c, and CD205 and expressed intermediate levels of CD86 and low to intermediate levels of CD14 with some donor-to-donor variation, as assessed by flow cytometry on an LSR-II flow cytometer (BD BioSciences) or an Attune NxT flow cytometer (Thermo

384 Fisher Scientific). All manipulations were performed in polystyrene conical tubes.

All experiments reported in this manuscript were conducted using the methods described above. Preliminary experiments were also performed after isolation of monocytes using the EasySep human monocyte enrichment kit with CD16 depletion (StemCell Technologies) and the EasySep human monocyte isolation kit (StemCell Technologies) with equivalent results.

389 Analogous experiments were also performed with cells cultured in RPMI 1640 medium without 390 supplementation of penicillin/ streptomycin/L-glutamine, with equivalent results. RNA-seq data

391 from moDCs from the same donor differentiated in parallel with and without

392 penicillin/streptomycin/L-glutamine were virtually identical (not shown).

Harvesting of moDCs

395 For all assays, both non-attached and loosely attached moDCs were harvested and then

396 combined. The culture supernatant containing the non-attached cells was first transferred to a

397 conical tube. The remaining attached cells were then detached by addition of CellStripper

398 (Corning, Corning, NY), a non-enzymatic dissociation solution, to the flask (3 mL for a T-150

399 flask, $1.5 \mathrm{~mL}$ for a T-75 flask, $0.5 \mathrm{~mL}$ for a T-25 flask) and incubation at $37{ }^{\circ} \mathrm{C}$ and $5 \% \mathrm{CO}_{2}$ for

$40015 \mathrm{~min}$. The cells were further detached by pipetting and gently tapping the flasks. The

401 suspension was aspirated into a new conical tube and another round of detachment with 
CellStripper was performed for $5 \mathrm{~min}$. The detached cells were combined, centrifuged at $100 \times \mathrm{g}$

403 for 10 min, resuspended in RPMI medium, and combined with the non-attached cells. Cell

404 counts were determined in duplicate using a Countess II automated hemocytometer (Thermo

405 Fisher Scientific); at least two squares were counted for each replicate. All manipulations were

406 performed in polystyrene conical tubes.

Treatments of moDCs

409 To prepare moDCs for treatments, an aliquot of cells containing an appropriate cell number was

410 centrifuged at $100 \times g$ for $10 \mathrm{~min}$. The cells were resuspended in complete RPMI medium

411 without cytokines. For readout by ELISA, cells were dispensed into flat-bottom 96-well plates in

412 aliquots of 20,000 cells in $200 \mu \mathrm{L}$ and incubated at $37^{\circ} \mathrm{C}$ and $5 \% \mathrm{CO}_{2}$ for $2-4 \mathrm{~h}$. Each

413 experiment contained medium-only (no cells) and PBS treatment (unstimulated/no treatment

414 control) negative controls. For subsequent RNA isolation, cells were dispensed into flat-bottom

415 24-well plates in aliquots of 200,000 to 250,000 cells at $1 \cdot 10^{6}$ cells $\cdot \mathrm{mL}^{-1}$, as indicated for each

416 experiment, and incubated at $37{ }^{\circ} \mathrm{C}$ and $5 \% \mathrm{CO}_{2}$ for $2-4 \mathrm{~h}$. To initiate the stimulation, purified

417 LPS or PBS (no treatment control) were added to each well to the final desired concentration.

418 LPS stocks were generally prepared at a 20x concentration such that all wells received an

419 equivalent volume of stimulant.

$420 \quad$ For readout by ELISA, the cells were incubated with the stimuli at $37{ }^{\circ} \mathrm{C}$ and $5 \% \mathrm{CO}_{2}$ for

$42120 \mathrm{~h}$, at which point the supernatants were transferred into a V-bottom 96-well plate, centrifuged

422 at $3200 \times g$ for 10 min to remove residual bacteria and cell debris, transferred to new plates, and

423 frozen at $-30{ }^{\circ} \mathrm{C}$ in aliquots. 
For RNA purifications, the cells were incubated with the stimuli at $37{ }^{\circ} \mathrm{C}$ and $5 \% \mathrm{CO}_{2}$ for

$4252 \mathrm{~h}$. To harvest RNA from treated cells, a 3x volume of TRIzol ${ }^{\mathrm{TM}}$ LS reagent (Ambion,

426 Naugatuck, CT) or TRI Reagent ${ }^{\circledR}$ (Zymo Research, Irvine, CA) was added directly to the cells.

427 The suspension was mixed by pipetting to lyse the cells, followed by RNA isolation using the

428 Direct-zol ${ }^{\mathrm{TM}}$ RNA Miniprep kit (Zymo Research) including an on-column DNase I digestion

429 step. Purified RNA was quantified using a Qubit Fluorometer (Thermo Fisher Scientific) and

430 stored at $-80^{\circ} \mathrm{C}$ until use.

432 Quantification of cytokine concentrations by ELISA

433 TNF- $\alpha$ concentrations in undiluted or appropriately diluted supernatants were determined by

434 ELISA using the SimpleStep human TNF alpha ELISA kit (abcam, Cambridge, MA), performed

435 following the manufacturer's instructions and with endpoint absorbance measurements at 450

$436 \mathrm{~nm}$ on an Infinite M200 Pro plate reader (Tecan, Männedorf, Switzerland). For each experiment,

437 absorbance measurements from wells containing a 2-fold dilution series of purified TNF- $\alpha$

438 (31.25 pg/mL to $2,000 \mathrm{pg} / \mathrm{mL}$, in duplicate) were used to calculate a calibration curve using a 4-

439 parameter logistic fit, which in turn was used to calculate TNF- $\alpha$ concentrations in all sample

440 wells. Concentrations of CXCL10 and IL-10 were determined equivalently using SimpleStep

441 human IP-10 ELISA kit (abcam) and the SimpleStep human IL-10 ELISA kit (abcam),

442 respectively, following the manufacturer's instructions. When handling multiple 96-well plates

443 simultaneously, plates were staggered in 3 min intervals starting with the last wash step to ensure

444 that incubation times with the development solution and stop solution were constant. 
448 To generate cDNA, purified RNA was reverse-transcribed using SuperScript ${ }^{\mathrm{TM}}$ III Reverse

449 Transcriptase (Thermo Fisher Scientific) with oligo(dT) primers in the presence of RNaseOUT ${ }^{\mathrm{TM}}$

450 Recombinant Ribonuclease Inhibitor (Thermo Fisher Scientific) or using SuperScript ${ }^{\mathrm{TM}}$ IV

451 VILO ${ }^{\mathrm{TM}}$ Master Mix (Thermo Fisher Scientific). All reactions in a given experiment were

452 normalized to contain the same amount of RNA (250 ng to $600 \mathrm{ng}$ depending on the

453 experiment). cDNA was diluted $1: 10$ and stored at $-30{ }^{\circ} \mathrm{C}$ until use. $\mathrm{qPCR}$ was performed using

454 the KAPA SYBR ${ }^{\circledR}$ FAST qPCR Master Mix (Roche, Basel, Switzerland) in $20 \mu L$ reactions

455 containing $3 \mu \mathrm{L}$ diluted cDNA and $200 \mathrm{nM}$ of each primer. Reactions were run on a

456 LightCycler ${ }^{\circledR} 480$ Instrument (Roche). All reactions were performed in technical triplicates. RT-

457 qPCR primers were chosen as intron-spanning primers, when possible, from the Universal

458 ProbeLibrary (Roche), with the following sequences:

459 ACTB: GCTACGAGCTGCCTGACG (fw), GGCTGGAAGAGTGCCTCA (rv)

460 IFNB 1: CTTTGCTATTTTCAGACAAGATTCA (fw), GCCAGGAGGTTCTCAACAAT (rv)

461 TNF: CAGCCTCTTCTCCTTCCTGAT (fw), GCCAGAGGGCTGATTAGAGA (rv)

462 CXCL10: GAAAGCAGTTAGCAAGGAAAGGT (fw),

463 GACATATACTCCATGTAGGGAAGTGA (rv)

Preparation of sequencing libraries for RNA-seq and data analysis

466 RNA-seq libraries were prepared from purified RNA using the Stranded mRNA Prep Ligation

467 kit (Illumina, San Diego, CA) in 96-well format, following the manufacturer's instructions. Input

468 RNA amounts were held constant for all samples for a given donor, between 300 and 600 ng per

469 reaction depending on the experiment. Final libraries were validated and quantified using the 
2100 Bioanalyzer (Agilent, Santa Clara, CA) using the High Sensitivity DNA kit (Agilent).

471 Paired-end 100 or paired-end 150 sequencing was performed on a HiSeq 4000 (Illumina). Reads

472 were aligned strand-specifically to the human genome (GRCh38) using the spliced read aligner

473 STAR (Dobin et al., 2013), version 2.6.0, against an index containing features from Gencode

474 release 34. Quantification of gene counts was carried out with featureCounts (Liao et al., 2014),

475 version 1.6.2, using only uniquely mapped reads to the reverse strand. Differential expression

476 analysis was carried out on gene counts using DESeq2 (Love et al., 2014), including only genes

477 with an average count $>2$ across all conditions. Transcript counts per million were calculated by

478 dividing gene counts by effective transcript length, using transcript length from Gencode

479 annotations and an average fragmenth length of 160 (the expected fragment size from the kit and

480 consistent with estimates determined by Kallisto (Bray et al., 2016)), followed by normalization

481 to total transcript counts. All other analyses were performed in python3.6.

$\operatorname{sgRNA}$ sequences

484 For initial experiments, an sgRNA sequence for $A A V S 1$ was chosen from a previous report (Mali 485 et al., 2013) and sgRNA sequences for TNF and TLR4 were chosen as the top predicted guides

486 from the Brunello library (Doench et al., 2016). All other sgRNAs were purchased from or

487 provided by Synthego (Menlo Park, CA), designed according to their multi-guide RNA strategy

488 (Stoner et al., 2019). Briefly, two or three sgRNAs are bioinformatically designed to work in a

489 cooperative manner to generate small, knockout-causing, fragment deletions in early exons.

490 These fragment deletions are larger than standard indels generated from single guides. The

491 genomic repair patterns from a multi-guide approach are highly predictable based on the guide-

492 spacing and design constraints to limit off-targets, resulting in a higher probability protein 
493 knockout phenotype. For the genetic screen, a Pattern Recognition Receptors and Signaling

494 Pathway arrayed library was provided by Synthego. All sgRNA sequences used in this

495 manuscript are listed in Supplementary File 1.

RNP assembly

498 RNPs were assembled by complexing purified recombinant Cas9 from Streptococcus pyogenes

499 (Synthego) with chemically synthesized sgRNAs (Synthego). Lyophilized sgRNAs targeting

500 each gene (individual or multiple sgRNAs) were resuspended to $100 \mu \mathrm{M}$ (total sgRNA

501 concentration) in RNase-free TE buffer (10 mM Tris, $1 \mathrm{mM} \mathrm{EDTA,} \mathrm{pH} \mathrm{8)} \mathrm{for} 15 \mathrm{~min}$ at $25^{\circ} \mathrm{C}$ or

502 over night at $4{ }^{\circ} \mathrm{C}$ with intermittent vortexing. Prior to use, sgRNA stocks were diluted to $25 \mu \mathrm{M}$

503 in RNase-free $\mathrm{H}_{2} \mathrm{O}$. Both stocks were stored at $-30{ }^{\circ} \mathrm{C}$ and freeze-thawed up to 5 times. To

504 assemble RNP for electroporation of $4 \cdot 10^{5}$ cells, 50 pmol sgRNA and 20 pmol Cas9 were

505 combined and diluted to $20 \mu \mathrm{L}$ with nucleofection solution P1 or P3 (with supplement added,

506 Lonza, Basel, Switzerland). The mixture was incubated at $25^{\circ} \mathrm{C}$ for $10 \mathrm{~min}$ or up to $2 \mathrm{~h}$ and

507 immediately used to electroporate moDCs. For double knockouts, 50 pmol of sgRNA against

508 each gene and 40 pmol Cas9 were combined in a total volume of $23 \mu \mathrm{L}$.

For experiments with guide RNAs in the crRNA:tracrRNA format (Figure 1 - Figure

510 Supplement 1d), lyophilized crRNAs and tracrRNA (Integrated DNA Technologies, Coralville,

511 Iowa) were resuspended to $400 \mu \mathrm{M}$ and $100 \mu \mathrm{M}$, respectively, in RNase-free TE buffer.

512 crRNA:tracrRNA duplexes were generated by mixing equimolar amounts of crRNA and

513 tracrRNA at a final concentration of $50 \mu \mathrm{M}$ each, incubating the mixture at $95^{\circ} \mathrm{C}$ for 5 min, and

514 allowing the mixture to cool to room temperature on the benchtop. To assemble RNP for

515 electroporation of $4 \cdot 10^{5}$ cells, 50 pmol crRNA:tracrRNA duplex and 20 pmol Cas9 v3 
516 (Integrated DNA Technologies, stock diluted to $20 \mu \mathrm{M}$ in sterile PBS) were combined and

517 diluted to $5 \mu \mathrm{L}$ in PBS, following the manufacturer's instructions.

519 moDC genome editing by electroporation of Cas 9 RNPS

520 Genome editing was performed by electroporation of moDCs with pre-formed Cas9 RNPs.

521 moDCs were detached as described above. A suspension containing an appropriate number of

522 moDCs $\left(4 \cdot 10^{5}\right.$ cells per electroporation $+5 \%$ excess $)$ was transferred to a new conical tube and

523 centrifuged at $90 \times \mathrm{g}$ for $10 \mathrm{~min}$. The cells were resuspended in $1-5 \mathrm{~mL}$ PBS and centrifuged

524 again at $90 \times \mathrm{g}$ for $10 \mathrm{~min}$. For electroporation with RNPs assembled with sgRNAs (all

525 experiments in this paper except for Figure 1 - Figure Supplement 1d, right), the cells were

526 resuspended in $5 \mu \mathrm{L}$ nucleofection solution P1 or P3 (with supplement added, Lonza) per $4 \cdot 10^{5}$

527 cells. Aliquots of $4 \cdot 10^{5}$ cells were transferred into individual wells of 16 -well or 96-well

528 nucleofection cuvettes (Lonza), combined with $20 \mu \mathrm{L}$ pre-formed RNP or nucleofector solution

529 (no RNP control), and immediately electroporated using pulse code DJ-108 (solution P3) or

530 other pulse codes, as described, using a Nucleofector-4D (Lonza) or a 96-well shuttle

531 (Amaxa/Lonza) attached to a Nuclefector-4D. For electroporation with RNPs assembled with

532 crRNA:tracrRNA duplexes Figure 1 - Figure Supplement 1d, right), the cells were resuspended

533 in $20 \mu \mathrm{L}$ nucleofection solution P1 or P3 (with supplement added, Lonza) per $4 \cdot 10^{5}$ cells and 5

$534 \mu \mathrm{L}$ pre-formed RNP or nucleofector solution (no RNP control) was added. Immediately after

535 nucleofection, $75 \mu \mathrm{L}$ pre-warmed complete RPMI medium supplemented with $50 \mathrm{ng} / \mathrm{mL}$ GM-

$536 \mathrm{CSF}$ and $20 \mathrm{ng} / \mathrm{mL}$ IL-4 were added to each well without disturbing the cells by letting the

537 medium run down the side of the cuvette. After incubation at $37^{\circ} \mathrm{C}$ and $5 \% \mathrm{CO}_{2}$ for $1 \mathrm{~h}$, the cells

538 were mixed by pipetting and then split into 2 wells of a flat-bottom 96-well plate filled with 50 
$\mu \mathrm{L}$ pre-warmed complete RPMI medium supplemented with $50 \mathrm{ng} / \mathrm{mL}$ GM-CSF and $20 \mathrm{ng} / \mathrm{mL}$

IL-4. The cells were incubated at $37{ }^{\circ} \mathrm{C}$ and $5 \% \mathrm{CO}_{2}$ for $5 \mathrm{~d}$, with medium replenished after 2 or $3 \mathrm{~d}$ and then used for assays.

543 electroporated with 10 pmol Cas9 and 25 pmol sgRNA) was obtained through iterative grid

544 searches of different conditions. In the process, several other conditions were also found to yield

545 good results, including nucleofection solution P1 with pulse code CB-128 (Figure 1 - Figure

546 Supplement 3a). In initial experiments, we also used solution P1 with pulse code CB-150 (Figure

547 1c, Figure 1 - Figure Supplement 1). Reducing the amount of Cas9 RNP led to a reduction in

548 editing efficiency.

551 For all experiments in this manuscript, electroporated moDCs were harvested for phenotyping

552 and genotyping $5 \mathrm{~d}$ post-electroporation, with the exception of data presented in Figure 1 -

553 Figure Supplement 1a, for which some moDCs were harvested 3 d post-electroporation. Both

554 non-attached and attached cells were harvested and then combined, largely as described above.

555 Briefly, the culture supernatants containing non-attached cells were first transferred to V-bottom

556 96-well plates. The remaining attached cells were then detached by addition of $25 \mu \mathrm{L}$

557 CellStripper solution (Corning) per well and incubation at $37^{\circ} \mathrm{C}$ and $5 \% \mathrm{CO}_{2}$ for $15 \mathrm{~min}$. The

558 cells were further detached by gentle pipetting and tapping of the plate and the suspension was

559 combined with the cell supernatant. Another round of detachment with CellStripper was

560 performed for $5 \mathrm{~min}$ and the suspension was combined with the suspension containing the

561 remaining cells. The cells suspensions were centrifuged at $100 \times \mathrm{g}$ for $10 \mathrm{~min}$ and resuspended in 
$562100 \mu \mathrm{L}$ complete RPMI medium without cytokines. Cells with the same knockout were

563 combined (cells from each electroporation had been split over 2 separate wells of a 96-well

564 plate) and used for phenotyping and genotyping.

565 To determine the responses of cells to stimuli by ELISA, aliquots of cells were

566 transferred into flat bottom 96-well plates, diluted to $200 \mu \mathrm{L}$ with complete RPMI medium

567 without cytokines, incubated at $37{ }^{\circ} \mathrm{C}$ and $5 \% \mathrm{CO}_{2}$ for $2-3 \mathrm{~h}$, and stimulated as described

568 above. Each stimulation was performed in duplicate. Supernatants from stimulated cells were

569 harvested and used to measure TNF- $\alpha$ levels as described above.

$570 \quad$ For subsequent RNA isolation, aliquots containing $1 \cdot 10^{5}$ to $2.5 \cdot 10^{5}$ cells were

571 transferred into flat-bottom 24 -well plates, diluted to $250 \mu \mathrm{L}$ with complete RPMI medium

572 without cytokines, incubated at $37{ }^{\circ} \mathrm{C}$ and $5 \% \mathrm{CO}_{2}$ for $2-3 \mathrm{~h}$, and stimulated as described

573 above. Each stimulation was performed in duplicate. RNA was extracted from treated cells as

574 described above.

575 During the incubation prior to stimulation, aliquots of the remaining cell suspension were

576 used to determine cell counts for each sample using a CellTiterGlo luminescence assay

577 (Promega, Madison, WI). Briefly, replicate aliquots of cells were transferred into an opaque flat-

578 bottom 96 -well plate, diluted to $100 \mu \mathrm{L}$, and incubated at $25^{\circ} \mathrm{C}$ for $15-30 \mathrm{~min}$. After addition

579 of an equal volume of CellTiterGlo solution to each well, the plates were placed on an orbital

580 shaker for $2 \mathrm{~min}$ and then incubated at $25{ }^{\circ} \mathrm{C}$ for $10 \mathrm{~min}$. Finally, luminescence in each well was

581 recorded using a GloMax Multi+ luminescence plate reader (Promega). For some experiments,

582 luminescence measurements from wells containing known numbers of unedited moDCs, as

583 determined using a Countess II automated hemocytometer (Thermo Fisher Scientific), were used

584 to calculate cell numbers for each sample. TNF- $\alpha$ secretion for each sample was then normalized 
to cell numbers. For other experiments, TNF- $\alpha$ secretion was simply normalized to backgroundsubtracted luminescence readings. In benchmark experiments, cell counts were also determined by flow cytometry on an LSR-II flow cytometer (BD Bioscences) equipped with a 96-well autosampler. Cell counts determined by flow cytometry and luminescence were well-correlated (Figure 1 - Figure Supplement 1a) and all further cell counts were determined by luminscence.

To isolate genomic DNA from each sample for genotyping, aliquots of harvested moDCs were transferred to a 96 -well V-bottom plate, centrifuged at $300 \times \mathrm{g}$ for $10 \mathrm{~min}$, and resuspended in $50 \mu \mathrm{L}$ QuickExtract DNA extraction solution (LuciGen, Middleton, WI). The suspensions were transferred to 96 -well PCR plates and incubated at $65^{\circ} \mathrm{C}$ for $20 \mathrm{~min}$ and then at $98^{\circ} \mathrm{C}$ for 5 min using a thermocycler. The extracted genomic DNA was stored at $-30{ }^{\circ} \mathrm{C}$ until use.

597 Genomic regions surrounding each cut site were PCR-amplified using a 2-step protocol, largely 598 as described (Leenay et al., 2019). Briefly, primer pairs were designed for each cut site using 599 Primer-BLAST (Ye et al., 2012) to amplify a 200- to 450-base pair region, ensuring that all cut 600 sites targeted by the pooled sgRNAs as well as a 50-base pair flanking region on each side of the

601 cut sites were included, with a targeted $\mathrm{T}_{\mathrm{m}}$ of $60^{\circ} \mathrm{C}$. Constant adapters (forward: $5^{\prime}-$

602 CTCTTTCCCTACACGACGCTCTTCCGATCT-3'; reverse: 5'-

603 CTGGAGTTCAGACGTGTGCTCTTCCGATCT-3') were appended to the designed primer

604 pairs. First-round PCRs of targeted sites were performed in 96-well format using at least 4,000

605 genomic copies for each sample, $0.5 \mu \mathrm{M}$ of each primer, and Q5 Hot Start High-Fidelity 2x

606 master mix (NEB, Ipswich, MA) and the following protocol: $98^{\circ} \mathrm{C}$ for $30 \mathrm{~s} ; 35$ cycles of $98^{\circ} \mathrm{C}$

607 for $10 \mathrm{~s}, 60{ }^{\circ} \mathrm{C}$ for $30 \mathrm{~s}$, and $72{ }^{\circ} \mathrm{C}$ for $30 \mathrm{~s}$; and a final extension at $72{ }^{\circ} \mathrm{C}$ for 2 min. Products 
608 from the first PCR were diluted 1:100 in $\mathrm{ddH}_{2} \mathrm{O}$ and subjected to a second round of PCR using

609 the constant adapters as annealing sites, appending Illumina P5 and P7 adapters and two eight-

610 base barcodes on both ends that together uniquely identified each sample. 12 cycles of PCR were

611 performed using the same conditions described above. After the second PCR, all samples were

612 pooled and the combined samples were purified using a 0.8x AMPure XP purification (Beckman

613 Coulter, Brea, CA). Final libraries were validated and quantified using the 2100 Bioanalyzer

614 (Agilent) using the High Sensitivity DNA kit (Agilent) and sequenced in a 600-cycle paired-end

615 run on a MiSeq Instrument (Illumina) using MiSeq v3 Reagent kits.

616 Sequencing data of editing outcomes were analyzed and quantified using knock-knock

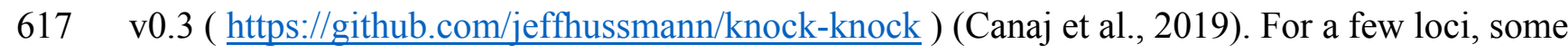

618 amplicons contained large deletions with boundaries $>20 \mathrm{bp}$ from an sgRNA cut site that were

619 classified as "large deletions" by knock-knock but likely instead reflect amplification of partially

620 complementary fragments, given in particular the rare occurrence of large deletions with

621 individual sgRNAs. To avoid overestimating editing efficiency, reads with at least one alignment

622 boundary $>40 \mathrm{bp}$ from an sgRNA cut site or both alignment boundaries $>20$ bp from sgRNA cut

623 sites were re-classified into the "malformed layout" category. For all experiments in which a

624 gene was targeted with multiple sgRNAs, sequencing counts were adjusted by the size difference

625 to the WT locus, using the following formula:

$$
\text { count }_{\text {corr }}=\text { count } * 2^{-0.014356 *\left(l_{W T}-l_{\text {read }}\right)}
$$

627 where count is the raw count, lwT the length in bp of the WT locus, and 1read the length in bp of

628 the edited locus. See below for a description of how the coefficient was derived. Results from

629 outcome classification, after correction for size, for all experiments except for the screen are 
630 listed in Supplementary File 2. Results for the screen, after correction for size, are listed in

631 Supplementary File 4.

633 Empirical assessment of amplicon size bias

634 To measure how amplicon size affects amplification and sequencing efficiency in our genotyping

635 approach, we subjected pools of purified amplicons of defined sizes to our sequencing library

636 preparation protocol and determined the resulting sequencing counts (Figure 1 - Figure

637 Supplement 2a). Briefly, we designed the strategy based on the following criteria:

638 1) Amplicons are of defined sizes between $\sim 150 \mathrm{bp}$ and $\sim 500 \mathrm{bp}$ (the range of amplicon

639 sizes in our experiments) and amplified by the same primer pair;

640 2) Sequencing library preparation protocol is analogous to that used for genotyping,

645 Briefly, we generated five amplicons with final lengths of 146 bp, 249 bp, 349 bp, 447 bp, and

$646539 \mathrm{bp}$ and with constant annealing sites at the ends by PCR-amplifying different fragments of a

647 gene encoding BFP with a constant forward primer and reverse primers positioned at the

648 appropriate distances. Forward and reverse primers contained overhangs (identical for all reverse

649 primers) to create annealing sites for sequencing library preparation. Following the PCR, each

650 individual amplicon was gel-purified and quantified using a Qubit Fluorometer (Thermo Fisher

651 Scientific). The five amplicons were then mixed into pools at 11 different molar ratios. For

652 increased accuracy, the abundance of each fragment in these pools was measured using the 2100 
653 Bioanalyzer (Agilent) using the High Sensitivity DNA kit (Agilent). Each pool was then diluted

654 to $33 \mathrm{fM}$ (about 20,000 template molecules per $\mu \mathrm{L}$, equivalent to genomic DNA isolated from

65510,000 cells) and $1 \mu \mathrm{L}$ of diluted pool was used as template for the first-round PCR as described

656 above, using a primer pair complementary to the constant overhangs on each fragment, designed

657 with the same criteria as our other amplicon primers. The PCRs additionally contained $4 \mu \mathrm{L}$ of

658 genomic DNA from unedited DCs as excess non-productive template. The remainder of the

659 sequencing library preparation was carried out as described above, with unique sequencing

660 indices appended to each pool in the second-round PCR. The final libraries were sequenced on a

661 MiSeq (Illumina) and counts for each fragment were determined by aligning reads to the

662 expected amplicons.

663 To infer observation efficiency (amplification + sequencing) for each fragment, we

664 reasoned that the starting and the final composition of the pool should be related by the specific

665 observation efficiency of each amplicon, which should shift the abundance of each fragment

666 based on its specific observation efficiency:

$$
m_{i}=\frac{e_{i} s_{i}}{\sum_{j=1}^{n} e_{j} s_{j}}
$$

668 where $m_{i}$ is the measured fractional abundance, $s_{i}$ the starting abundance, and $e_{i}$ the fragment-

669 specific observation efficiency. Note that the equation takes this form because we can measure

670 only fractional rather than absolute abundances of each amplicon at the end; thus

$$
\sum_{j=1}^{n} m_{j}=1
$$

672 To compute the efficiencies, we arbitrarily set the efficiency e of the $447 \mathrm{bp}$ fragment (which

673 was included in all pools) to $1\left(e_{n}=1\right)$ and then solved the resulting linear equation system

$$
m_{i} \sum_{j=1}^{n} e_{j} s_{j}=e_{i} s_{i}
$$




$$
\left(m_{i}-1\right) e_{i} s_{i}+m_{i} \sum_{j \neq i}^{n-1} e_{j} s_{j}=-m_{i} s_{n}
$$

676 to obtain the observation efficiencies (amplification + sequencing) $e_{i}$ for each fragment in each

677 pool. Because we expected per-cycle PCR amplification efficiency to be a major contributor to

678 these efficiencies, we compared $\log _{2}$ e to fragment size and found it to be linearly correlated

679 (Figure 1 - Figure Supplement 2b). We therefore estimated the contribution of each bp in size

680 difference to observation efficiency using a linear regression of $\log _{2}$ e against length in bp; we

681 used the slope of this regression to correct sequencing counts as described above. We note that

682 size bias appears to be less evident when small amplicons are already overrepresented in the

683 input (Figure 1 - Figure Supplement 2b), perhaps because under these conditions primers rather

684 than nucleotides are the limiting component in PCR. Because our editing efficiencies are

685 generally high and thus smaller fragments are more abundant at the outset than longer fragments,

686 our correction approach is (intentionally) conservative and should not cause us to overestimate

687 editing efficiency.

690 RNA-seq libraries were prepared from purified RNA as described above. Input RNA amounts

691 were held constant for all samples for a given donor (250 ng for donor p and $400 \mathrm{ng}$ for donor q).

692 For donor $\mathrm{p}$, the RNA extraction for one replicate sample with knockout of RPE65 and treated

693 with $100 \mathrm{ng} / \mathrm{mL}$ B. theta LPS failed. Reads were aligned strand-specifically to the human

694 genome, gene counts were quantified, and differential gene expression analysis was conducted as

695 described above. Read coverage along transcripts was quantified using plastid (Dunn and

696 Weissman, 2016). All other analyses were performed in python3.6. 
699 To select genes to target in our arrayed genetic screen, we first included all genes from the

700 following categories of pattern recognition receptors (PRRs): Toll-like receptors, NOD-like

701 receptors, RIG-I-like receptors, C-type lectin receptors, Galectins, and SIGLECs. We then

702 assembled a list of all genes encoding relevant signaling proteins downstream of these PRRs,

703 including immediate adaptor proteins, kinases and ubiquitin ligases, the downstream

704 transcription factors, as well a limited subset of effector cytokines and cytokine receptors.

705 Finally, we completed the gene list with additional genes of interest by surveying our RNA-seq

706 data from human moDCs for expressed potential pattern recognition receptors such as predicted

707 surface/membrane proteins, carbohydrate-binding proteins, and proteins containing a V-set

708 domain using searches for Pfam domains, and by browsing the list of genes with the GO term

709 "innate immune response" that we had not yet included. This process ultimately resulted in a list

710 of $>400$ genes. To narrow the list down to $\sim 300$ genes to enable screening in four 96-well plates,

711 we first eliminated pseudogenes and a few PRR-like genes with well-established functions. We

712 then eliminated many genes involved in linear signaling pathways while ensuring that each

713 pathway was targeted at multiple nodes in the final library. In total, we targeted 291 unique

714 genes of interest.

715 The library additionally included 4 classes of controls: 1) non-targeting negative controls;

716 2) neutral controls (targeting negative controls); 3) targeting positive controls; and 4) essential

717 controls. As non-targeting negative controls, we picked non-targeting negative controls \#1 and

718 \#2 from Synthego. As neutral controls, we selected 9 genes (CRX, KCNV1, TRHR, LALBA,

719 RPE65, F13B, OR2D2, OR51T1, TAS2R9) that are not expressed in moDCs, as assessed by our

720 RNA-seq data and non-essential in any cell type surveyed at the time (from Project Achilles) 
721 (Tsherniak et al., 2017), and for which some functional annotation existed such as tissue-specific

722 activity. These genes include olfactory and taste receptors as well as genes expressed only in

723 specific tissues such as the retinal pigment epithelium or the testes. As a targeting positive

724 control for TNF- $\alpha$ ELISA readout, we included TNF. As essential controls, we included the two

725 core essential genes (Hart et al., 2015): U2AF2 (a splicing factor) and POLR2A (a component of

726 RNA polymerase II). In total, we included 14 controls. All sgRNA sequences are listed in

727 Supplementary File 1.

728 Finally, all selected genes were arrayed into 96-well format, with the following design

729 principles: each of the four 96-well plates contained each of the 14 controls in randomized

730 positions on each plate; column 12 was left empty for no pulse electroporation controls and

731 media-only ELISA controls; the remaining 74 positions on each 96-well plate were randomly

732 filled with sgRNAs targeting genes of interest. The final plate layouts are depicted in Figure 3 -

733 Figure Supplement 1 and listed in Supplementary File 3.

736 The Pattern Recognition Receptors and Signaling Pathway arrayed library targeting all selected

737 genes with up to 3 sgRNAs per gene was provided by Synthego. For a few genes, high homology

738 to other loci precluded selection of 3 unique sgRNAs within a 200-bp window; in those cases 2

739 or 1 sgRNAs were used. Purified, lyophilized sgRNAs were resuspended to $25 \mu \mathrm{M}$ in $0.25 \mathrm{x}$ TE

740 for $16 \mathrm{~h}$ at $4{ }^{\circ} \mathrm{C}$, aliquotted into $96-w e l l$ plates, and frozen at $-80{ }^{\circ} \mathrm{C}$ until use.

$741 \quad$ For each screen, monocytes were isolated from $1.5 \cdot 10^{9}$ PBMCs (AllCells) from a single

742 healthy human donor and differentiated into moDCs as described above. Differentiated moDCs

743 were electroporated with sgRNAs in 96-well format as described above. Plates were staggered in 
74410 min intervals to minimize the amount of time cells spent in nucleofection solution and the

745 time delay between electroporation and addition of recovery media. On day 3 after

746 electroporation, 4 wells of cells containing no pulse/no RNP control cells were harvested to

747 assess responses of unedited cells to $B$. theta LPS and E. coli LPS and to determine an optimal $B$.

748 theta LPS concentration for treatment of edited cells (Figure 3e). Maintenance, harvesting, and

749 counting of electroporated moDCs was performed as described above. Plates were staggered for

750 luminescence reads to keep incubation time with the luminescence substrate constant. All cells

751 were treated with $100 \mathrm{ng} / \mathrm{mL}$ B. theta LPS in a volume of $200 \mu \mathrm{L}$. Concentrations of TNF- $\alpha$ and

752 IL-10 in cell supernatants were determined by ELISA as described above. Plates were staggered

753 in 7 min intervals throughout the entire process to keep incubation times constant.

$754 \quad \log _{2}$ fold-changes in TNF- $\alpha$ or IL-10 secretion were calculated as follows:

755 1) ELISA absorbance values for each individual sample were background-corrected

756 using absorbance values from media-only wells. For the IL-10 ELISAs, background-

757 corrected absorbance below 0 (recorded for four samples) were assigned the

758 background-corrected absorbance of the sample with the lowest value greater than 0.

759 2) Background-corrected absorbance values were normalized by the average

760 luminescence (average of two replicate measurements) for each cell sample to

761 calculate a cell count-normalized absorbance.

762 3) For each sample, the cell count-normalized absorbance was normalized to the median

763 cell count-normalized absorbance of all 9 neutral targeting controls on the same 96-

764 well plate to calculate a fold-change in TNF- $\alpha$ secretion. Normalization was

765 performed by plate to normalize for any plate effects. 
4) Fold-changes were $\log _{2}$ transformed to calculate $\log _{2}$ fold-changes in TNF- $\alpha$ secretion.

5) $\log _{2}$ fold changes of replicate treatments were averaged to calculate the average $\log _{2}$ fold change of each knockout population. Normality tests suggested that the $\log _{2}$ foldchanges were generally normally distributed, rationalizing the averaging of the $\log _{2}$ transformed values.

Raw and processed ELISA and cell count data are included in Supplementary File 3. maximum amplicon size of 500 base pairs and a minimum flanking distance of 25 base pairs.

781 Primers containing overhangs as described above were ordered in 96-well format matching the 782 sgRNA layout and tested for efficient amplification of the targeted locus by amplifying genomic

783 DNA from unedited moDCs and sequencing the resulting amplicons on a MiSeq (Illumina), as

784 described above. For loci with inefficient amplification or a high fraction of off-target amplicons, 785 as assessed using knock-knock (Canaj et al., 2019, p.), primers were designed using

786 PrimerBLAST (Ye et al., 2012) as described above, and efficient amplification confirmed by

787 sequencing. For some highly homologous locus pairs, such as SFTPA1/SFTPA2 and

$788 L G A L S 7 / L G A L S 7 B$, no primers could be designed that ensured completely unique amplification 
of each individual locus; the primers with the best discriminating power that fit all other design criteria (amplicon size, Tm, distance from cut site) were chosen. All primers are listed in

791 Supplementary File 1. as described above, with the following modifications: 1) PCRs were performed in 384-well

794 format; 2) first-round PCRs were set up using a Biomek FX liquid handling system with a 96well head (Beckman Coulter); 3) first-round PCR products were diluted into Echo Qualified 384Well Polypropylene Microplates using the Biomek FX; 4) PCR mastermix for the second-round PCR was dispensed into 384-well PCR plates using the Biomek FX; and 5) diluted first-round PCR products and indexing PCR primers were dispensed into the 384-well PCR plate using an Echo 525 acoustic liquid handler (Labcyte, San Jose, CA). Purification and validation of sequencing libraries, sequencing, classification of sequencing outcomes, and correction for

801 amplicon size were performed as described above. For donor h, a small set of samples did not 802 produce aligning sequencing reads in a first PCR attempt. These samples were repeated manually 803 as described above, after which all but one sample produced aligning sequencing reads. Only

804 successfully prepared samples were included for analysis. For donor i, the sequencing library

805 preparation was repeated independently for $>200$ loci, which produced near-identical results

806 (Figure 3 - Figure Supplement 4c), validating that the sequencing library preparation strategy is

807 robust and reproducible and that size-dependent amplification efficiency is consistent across

808 PCRs. Samples with $>100$ size-corrected on-target reads (generally corresponding to $>500$ raw

809 on-target reads) were included to estimate editing efficiencies. For some pairs of highly

810 homologous loci (e.g. SFTPA1 and SFTPA2), amplicons for both loci were detected with primer

811 pairs designed to amplify each individual locus because it was impossible to design completely 
812 specific primer pairs with the criteria used. These amplicons were not excluded when calculating

813 editing efficiency, such that editing efficiency is slightly underestimated for these loci. Results

814 from outcome classification, after correction for size, are listed in Supplementary File 4.

816 Sample sizes and sample size estimation

817 No sample-size calculation was performed in advance. All results were reproduced in cells from

818 multiple independent donors, following conventions of the field. Within independent

819 experiments, assays were performed in duplicate or triplicate following conventions of the field.

821 Replication and data exclusion

822 All main findings were derived from experiments with cells from at least 2 independent donors.

823 The main hits from the genetic screen were validated in cells from 2 additional, independent

824 donors. All treatments were performed in duplicate for readout by ELISA and qPCR and in

825 duplicate or triplicate for readout by RNA-seq. Cell counts were generally conducted in

826 duplicate. Information on number of replicates is contained in the figure legends.

827 For identification of differentially expressed genes in RNA-seq, only genes with an

828 average count $>2$ across all conditions were included for analysis. Exclusion criteria for editing

829 analysis are described in the corresponding methods sections.

\section{Data availability}

832 Raw data from RNA-seq of unedited and edited moDCs are available at GEO under accession 833 codes GSE161401 and GSE161466, respectively. Raw data from amplicon sequencing for all

834 samples are available at SRA under accession code PRJNA673198. Processed data from 
835 amplicon sequencing as well as raw and processed data from the genetic screens are provided as

836 supplemental files (Supplementary Files 2, 3, 4).

838 Code

839 Amplicon sequencing data were processed using the publicly available pipeline knock-knock (

840 https://github.com/jeffhussmann/knock-knock ) (Canaj et al., 2019). RNA-seq data were

841 processed using STAR (Dobin et al., 2013), featureCounts (Liao et al., 2014), and DESeq2 (Love

842 et al., 2014).

843

844 


\section{Acknowledgements}

846 We thank C. Gross, J. Hiatt, E. Chow (all UCSF), A. May (Chan-Zuckerberg Biohub), J. Kagan

847 (Boston Children's Hospital), J. Chen (UT Southwestern), G. Alberts (Lonza), and all members

848 of the Weissman, Fischbach, and Gross labs for helpful discussions, and E. Chow, D. Martinez,

849 and K. Chaung from the UCSF Center for Advanced Technology for help with sequencing. The

850 Pattern Recognition Receptors and Signaling Pathway arrayed library was provided by Synthego

851 as part of a collaboration agreement. B. theta LPS purifications were performed by Biswa P.

852 Choudhury at the UC San Diego GlycoAnalytics Core. JSW is a Howard Hughes Medical

853 Institute Investigator.

\section{Competing Interests}

856 JSW consults for and holds equity in KSQ Therapeutics, Maze Therapeutics, and Tenaya

857 Therapeutics. JSW is a venture partner at 5AM Ventures and a member of the Amgen Scientific

858 Advisory Board. MAF is a co-founder and director of Federation Bio and Viralogic. MJ consults

859 for Maze Therapeutics. JAH consults for Tessera Therapeutics.

860

861 


\section{References}

Antebi, Y.E., Linton, J.M., Klumpe, H., Bintu, B., Gong, M., Su, C., McCardell, R., Elowitz, M.B., 2017. Combinatorial Signal Perception in the BMP Pathway. Cell 170, 11841196.e24. https://doi.org/10.1016/j.cell.2017.08.015

Bray, N.L., Pimentel, H., Melsted, P., Pachter, L., 2016. Near-optimal probabilistic RNA-seq quantification. Nature Biotechnology 34, 525-527. https://doi.org/10.1038/nbt.3519

C. Khouili, S., Cook, E.C.L., Hernández-García, E., Martínez-López, M., Conde-Garrosa, R., Iborra, S., 2020. SHP-1 Regulates Antigen Cross-Presentation and Is Exploited by Leishmania to Evade Immunity. Cell Reports 33, 108468. https://doi.org/10.1016/j.celrep.2020.108468

Canaj, H., Hussmann, J.A., Li, H., Beckman, K.A., Goodrich, L., Cho, N.H., Li, Y.J., Santos, D.A., McGeever, A., Stewart, E.M., Pessino, V., Mandegar, M.A., Huang, C., Gan, L., Panning, B., Huang, B., Weissman, J.S., Leonetti, M.D., 2019. Deep profiling reveals substantial heterogeneity of integration outcomes in CRISPR knock-in experiments. bioRxiv 841098. https://doi.org/10.1101/841098

Coats, S.R., Berezow, A.B., To, T.T., Jain, S., Bainbridge, B.W., Banani, K.P., Darveau, R.P., 2011. The Lipid A Phosphate Position Determines Differential Host Toll-Like Receptor 4 Responses to Phylogenetically Related Symbiotic and Pathogenic Bacteria. Infection and Immunity 79, 203-210. https://doi.org/10.1128/IAI.00937-10

Cullen, T.W., Schofield, W.B., Barry, N.A., Putnam, E.E., Rundell, E.A., Trent, M.S., Degnan, P.H., Booth, C.J., Yu, H., Goodman, A.L., 2015. Antimicrobial peptide resistance mediates resilience of prominent gut commensals during inflammation. Science 347, 170-175. https://doi.org/10.1126/science. 1260580 
885

886

887

888

889

890

891

892

893

894

895

896

897

898

899

900

901

902

903

904

905

906

d'Hennezel, E., Abubucker, S., Murphy, L.O., Cullen, T.W., 2017. Total Lipopolysaccharide from the Human Gut Microbiome Silences Toll-Like Receptor Signaling. mSystems 2. https://doi.org/10.1128/mSystems.00046-17

Di Lorenzo, F., Pither, M.D., Martufi, M., Scarinci, I., Guzmán-Caldentey, J., Łakomiec, E., Jachymek, W., Bruijns, S.C.M., Santamaría, S.M., Frick, J.-S., van Kooyk, Y., Chiodo, F., Silipo, A., Bernardini, M.L., Molinaro, A., 2020. Pairing Bacteroides vulgatus LPS Structure with Its Immunomodulatory Effects on Human Cellular Models. ACS Cent. Sci. 6, 1602-1616. https://doi.org/10.1021/acscentsci.0c00791

Ding, Y., Guo, Z., Liu, Y., Li, X., Zhang, Q., Xu, X., Gu, Y., Zhang, Y., Zhao, D., Cao, X., 2016. The lectin Siglec-G inhibits dendritic cell cross-presentation by impairing MHC class I-peptide complex formation. Nature Immunology 17, 1167-1175. https://doi.org/10.1038/ni.3535

Dobin, A., Davis, C.A., Schlesinger, F., Drenkow, J., Zaleski, C., Jha, S., Batut, P., Chaisson, M., Gingeras, T.R., 2013. STAR: ultrafast universal RNA-seq aligner. Bioinformatics 29, 15-21. https://doi.org/10.1093/bioinformatics/bts635

Doench, J.G., Fusi, N., Sullender, M., Hegde, M., Vaimberg, E.W., Donovan, K.F., Smith, I., Tothova, Z., Wilen, C., Orchard, R., Virgin, H.W., Listgarten, J., Root, D.E., 2016. Optimized sgRNA design to maximize activity and minimize off-target effects of CRISPR-Cas9. Nature Biotechnology 34, 184-191. https://doi.org/10.1038/nbt.3437

Dunn, J.G., Weissman, J.S., 2016. Plastid: nucleotide-resolution analysis of next-generation sequencing and genomics data. BMC Genomics 17, 958. https://doi.org/10.1186/s12864016-3278-x 
907

908

909

910

911

912

913

914

915

916

917

918

919

920

921

922

923

924

925

926

927

928

Fitzgerald, K.A., Kagan, J.C., 2020. Toll-like Receptors and the Control of Immunity. Cell 180, 1044-1066. https://doi.org/10.1016/j.cell.2020.02.041

Fitzgerald, K.A., Palsson-McDermott, E.M., Bowie, A.G., Jefferies, C.A., Mansell, A.S., Brady, G., Brint, E., Dunne, A., Gray, P., Harte, M.T., McMurray, D., Smith, D.E., Sims, J.E., Bird, T.A., O’Neill, L.A.J., 2001. Mal (MyD88-adapter-like) is required for Toll-like receptor-4 signal transduction. Nature 413, 78-83. https://doi.org/10.1038/35092578

Fitzgerald, K.A., Rowe, D.C., Barnes, B.J., Caffrey, D.R., Visintin, A., Latz, E., Monks, B., Pitha, P.M., Golenbock, D.T., 2003. LPS-TLR4 Signaling to IRF-3/7 and NF-кB Involves the Toll Adapters TRAM and TRIF. J Exp Med 198, 1043-1055. https://doi.org/10.1084/jem.20031023

Freund, E.C., Lock, J.Y., Oh, J., Maculins, T., Delamarre, L., Bohlen, C.J., Haley, B., Murthy, A., 2020. Efficient gene knockout in primary human and murine myeloid cells by nonviral delivery of CRISPR-Cas9. J Exp Med 217. https://doi.org/10.1084/jem.20191692

Garg, A.D., Coulie, P.G., Van den Eynde, B.J., Agostinis, P., 2017. Integrating Next-Generation Dendritic Cell Vaccines into the Current Cancer Immunotherapy Landscape. Trends in Immunology 38, 577-593. https://doi.org/10.1016/j.it.2017.05.006

Golenbock, D.T., Hampton, R.Y., Qureshi, N., Takayama, K., Raetz, C.R., 1991. Lipid A-like molecules that antagonize the effects of endotoxins on human monocytes. J. Biol. Chem. 266, 19490-19498.

Hart, T., Chandrashekhar, M., Aregger, M., Steinhart, Z., Brown, K.R., MacLeod, G., Mis, M., Zimmermann, M., Fradet-Turcotte, A., Sun, S., Mero, P., Dirks, P., Sidhu, S., Roth, F.P., Rissland, O.S., Durocher, D., Angers, S., Moffat, J., 2015. High-Resolution CRISPR 
931 Hiatt, J., Cavero, D.A., McGregor, M.J., Gordon, D.E., Zheng, W., Budzik, J.M., Roth, T.L., CRISPR-Cas9 Ribonucleoproteins. bioRxiv 2020.03.13.991414.

Jacobson, A.N., Choudhury, B.P., Fischbach, M.A., 2018. The Biosynthesis of Lipooligosaccharide from Bacteroides thetaiotaomicron. mBio 9. https://doi.org/10.1128/mBio.02289-17

Kaelin, W.G., 2012. Use and Abuse of RNAi to Study Mammalian Gene Function. Science 337, 421-422. https://doi.org/10.1126/science.1225787 H.Q., Uldbjerg, N., Porteus, M., Roan, N.R., Nyegaard, M., Denton, P.W., Jakobsen,

947 Leenay, R.T., Aghazadeh, A., Hiatt, J., Tse, D., Roth, T.L., Apathy, R., Shifrut, E., Hultquist, 948 J.F., Krogan, N., Wu, Z., Cirolia, G., Canaj, H., Leonetti, M.D., Marson, A., May, A.P., 949 Zou, J., 2019. Large dataset enables prediction of repair after CRISPR-Cas9 editing in primary T cells. Nature Biotechnology 37, 1034-1037. https://doi.org/10.1038/s41587019-0203-2 
952

953

954

955

956

957

958

959

960

961

962

963

964

965

966

967

968

969

970

971

972

973

974

Liao, Y., Smyth, G.K., Shi, W., 2014. featureCounts: an efficient general purpose program for assigning sequence reads to genomic features. Bioinformatics 30, 923-930. https://doi.org/10.1093/bioinformatics/btt656

Love, M.I., Huber, W., Anders, S., 2014. Moderated estimation of fold change and dispersion for RNA-seq data with DESeq2. Genome Biology 15, 550. https://doi.org/10.1186/s13059014-0550-8

Lucas, C., Wong, P., Klein, J., Castro, T.B.R., Silva, J., Sundaram, M., Ellingson, M.K., Mao, T., Oh, J.E., Israelow, B., Takahashi, T., Tokuyama, M., Lu, P., Venkataraman, A., Park, A., Mohanty, S., Wang, H., Wyllie, A.L., Vogels, C.B.F., Earnest, R., Lapidus, S., Ott, I.M., Moore, A.J., Muenker, M.C., Fournier, J.B., Campbell, M., Odio, C.D., CasanovasMassana, A., Herbst, R., Shaw, A.C., Medzhitov, R., Schulz, W.L., Grubaugh, N.D., Cruz, C.D., Farhadian, S., Ko, A.I., Omer, S.B., Iwasaki, A., 2020. Longitudinal analyses reveal immunological misfiring in severe COVID-19. Nature 584, 463-469. https://doi.org/10.1038/s41586-020-2588-y

Mali, P., Yang, L., Esvelt, K.M., Aach, J., Guell, M., DiCarlo, J.E., Norville, J.E., Church, G.M., 2013. RNA-Guided Human Genome Engineering via Cas9. Science 339, 823-826. https://doi.org/10.1126/science.1232033

Merad, M., Sathe, P., Helft, J., Miller, J., Mortha, A., 2013. The Dendritic Cell Lineage: Ontogeny and Function of Dendritic Cells and Their Subsets in the Steady State and the Inflamed Setting. Annu. Rev. Immunol. 31, 563-604. https://doi.org/10.1146/annurevimmunol-020711-074950

Pereira, N.L., Ahmad, F., Cummins, N.W., Byku, M., Morris, A.A., Owens, A., Tuteja, S., Cresci, S., 2020. COVID-19: Understanding Inter-Individual Variability and Implications 
for Precision Medicine. Mayo Clinic Proceedings.

977 Poltorak, A., He, X., Smirnova, I., Liu, M.-Y., Huffel, C.V., Du, X., Birdwell, D., Alejos, E.,

978 Silva, M., Galanos, C., Freudenberg, M., Ricciardi-Castagnoli, P., Layton, B., Beutler, B., 1998. Defective LPS Signaling in C3H/HeJ and C57BL/10ScCr Mice: Mutations in Tlr4 Gene. Science 282, 2085-2088. https://doi.org/10.1126/science.282.5396.2085 https://doi.org/10.1016/j.chom.2017.08.020

985

986

Pulendran, B., Davis, M.M., 2020. The science and medicine of human immunology. Science 369. https://doi.org/10.1126/science.aay4014

Riggan, L., Hildreth, A.D., Rolot, M., Wong, Y.-Y., Satyadi, W., Sun, R., Huerta, C., O’Sullivan, T.E., 2020. CRISPR-Cas9 Ribonucleoprotein-Mediated Genomic Editing in Mature Primary Innate Immune Cells. Cell Reports 31, 107651. https://doi.org/10.1016/j.celrep.2020.107651

Roth, T.L., Puig-Saus, C., Yu, R., Shifrut, E., Carnevale, J., Li, P.J., Hiatt, J., Saco, J., Krystofinski, P., Li, H., Tobin, V., Nguyen, D.N., Lee, M.R., Putnam, A.L., Ferris, A.L., Chen, J.W., Schickel, J.-N., Pellerin, L., Carmody, D., Alkorta-Aranburu, G., del Gaudio, D., Matsumoto, H., Morell, M., Mao, Y., Cho, M., Quadros, R.M., Gurumurthy, C.B., Smith, B., Haugwitz, M., Hughes, S.H., Weissman, J.S., Schumann, K., Esensten, J.H., May, A.P., Ashworth, A., Kupfer, G.M., Greeley, S.A.W., Bacchetta, R., Meffre, E., Roncarolo, M.G., Romberg, N., Herold, K.C., Ribas, A., Leonetti, M.D., Marson, A., 
998

999

1000

1001

1002

1003

1004

1005

1006

1007

1008

1009

1010

1011

1012

1013

1014

1015

1016

1017

1018

1019

2018. Reprogramming human T cell function and specificity with non-viral genome targeting. Nature 559, 405-409. https://doi.org/10.1038/s41586-018-0326-5

Sallusto, F., Lanzavecchia, A., 1994. Efficient presentation of soluble antigen by cultured human dendritic cells is maintained by granulocyte/macrophage colony-stimulating factor plus interleukin 4 and downregulated by tumor necrosis factor alpha. J Exp Med 179, 11091118. https://doi.org/10.1084/jem.179.4.1109

Sanz, J., Randolph, H.E., Barreiro, L.B., 2018. Genetic and evolutionary determinants of human population variation in immune responses. Current Opinion in Genetics \& Development, Genetics of Human Origins 53, 28-35. https://doi.org/10.1016/j.gde.2018.06.009

Schumann, K., Lin, S., Boyer, E., Simeonov, D.R., Subramaniam, M., Gate, R.E., Haliburton, G.E., Ye, C.J., Bluestone, J.A., Doudna, J.A., Marson, A., 2015. Generation of knock-in primary human T cells using Cas9 ribonucleoproteins. PNAS 112, 10437-10442. https://doi.org/10.1073/pnas.1512503112

Song, X.-T., 2014. Genetic Modification of Dendritic Cells with RNAi, in: Lawman, M.J.P., Lawman, P.D. (Eds.), Cancer Vaccines: Methods and Protocols, Methods in Molecular Biology. Springer, New York, NY, pp. 119-130. https://doi.org/10.1007/978-1-49390345-0_11

Stefan, K.L., Kim, M.V., Iwasaki, A., Kasper, D.L., 2020. Commensal Microbiota Modulation of Natural Resistance to Virus Infection. Cell 0. https://doi.org/10.1016/j.cell.2020.10.047

Steimle, A., Michaelis, L., Di Lorenzo, F., Kliem, T., Münzner, T., Maerz, J.K., Schäfer, A., Lange, A., Parusel, R., Gronbach, K., Fuchs, K., Silipo, A., Öz, H.H., Pichler, B.J., Autenrieth, I.B., Molinaro, A., Frick, J.-S., 2019. Weak Agonistic LPS Restores Intestinal 
Immune Homeostasis. Molecular Therapy 27, 1974-1991.

https://doi.org/10.1016/j.ymthe.2019.07.007

1022 Stoner, R., Maures, T., Conant, D., 2019. METHODS AND SYSTEMS FOR GUIDE RNA DESIGN AND USE. U.S. Patent 2019/0382797 A1.

1024 Sun, T., Nguyen, A., Gommerman, J.L., 2020. Dendritic Cell Subsets in Intestinal Immunity and Inflammation. The Journal of Immunology 204, 1075-1083.

Tan, Y., Zanoni, I., Cullen, T.W., Goodman, A.L., Kagan, J.C., 2015. Mechanisms of Toll-like Y., Jiang, G., Hsiao, J., Gerath, W.F.J., Howell, S., Merkel, E., Ghandi, M., Garraway, 
1042 Wculek, S.K., Cueto, F.J., Mujal, A.M., Melero, I., Krummel, M.F., Sancho, D., 2020. Dendritic

1043

1044

1045

1046

1047

1048

1049

1050

1051

1052

1053

1054

1055

1056

1057

1058

1059

1060

1061

1062

1063

cells in cancer immunology and immunotherapy. Nature Reviews Immunology 20, 7-24. https://doi.org/10.1038/s41577-019-0210-z

Weintraub, A., Zähringer, U., Wollenweber, H.-W., Seydel, U., Rietschel, E.T., 1989. Structural characterization of the lipid A component of Bacteroides fragilis strain NCTC 9343 lipopolysaccharide. European Journal of Biochemistry 183, 425-431. https://doi.org/10.1111/j.1432-1033.1989.tb14945.x

Werts, C., Tapping, R.I., Mathison, J.C., Chuang, T.-H., Kravchenko, V., Saint Girons, I., Haake, D.A., Godowski, P.J., Hayashi, F., Ozinsky, A., Underhill, D.M., Kirschning, C.J., Wagner, H., Aderem, A., Tobias, P.S., Ulevitch, R.J., 2001. Leptospiral lipopolysaccharide activates cells through a TLR2-dependent mechanism. Nature Immunology 2, 346-352. https://doi.org/10.1038/86354

Wexler, A.G., Goodman, A.L., 2017. An insider's perspective: Bacteroides as a window into the microbiome. Nature Microbiology 2, 1-11. https://doi.org/10.1038/nmicrobiol.2017.26

Yamamoto, M., Sato, S., Hemmi, H., Hoshino, K., Kaisho, T., Sanjo, H., Takeuchi, O., Sugiyama, M., Okabe, M., Takeda, K., Akira, S., 2003. Role of Adaptor TRIF in the MyD88-Independent Toll-Like Receptor Signaling Pathway. Science 301, 640-643. https://doi.org/10.1126/science.1087262

Yamamoto, M., Sato, S., Mori, K., Hoshino, K., Takeuchi, O., Takeda, K., Akira, S., 2002. Cutting Edge: A Novel Toll/IL-1 Receptor Domain-Containing Adapter That Preferentially Activates the IFN- $\beta$ Promoter in the Toll-Like Receptor Signaling. The Journal of Immunology 169, 6668-6672. https://doi.org/10.4049/jimmunol.169.12.6668 
1064 Ye, J., Coulouris, G., Zaretskaya, I., Cutcutache, I., Rozen, S., Madden, T.L., 2012. Primer-

1065 BLAST: A tool to design target-specific primers for polymerase chain reaction. BMC

1066 Bioinformatics 13, 134. https://doi.org/10.1186/1471-2105-13-134

1067 Zanoni, I., Ostuni, R., Marek, L.R., Barresi, S., Barbalat, R., Barton, G.M., Granucci, F., Kagan,

1068 J.C., 2011. CD14 Controls the LPS-Induced Endocytosis of Toll-like Receptor 4. Cell 147, 868-880. https://doi.org/10.1016/j.cell.2011.09.051

1070 Zhu, T., Liang, C., Meng, Z., Li, Y., Wu, Y., Guo, S., Zhang, R., 2017. PrimerServer: a high1071 throughput primer design and specificity-checking platform. bioRxiv 181941.

$1072 \quad$ https://doi.org/10.1101/181941

1073

1074 
bioRxiv preprint doi: https://doi.org/10.1101/2020.12.22.423985; this version posted December 22, 2020. The copyright holder for this preprint (which was not certified by peer review) is the author/funder, who has granted bioRxiv a license to display the preprint in perpetuity. It is made available under aCC-BY 4.0 International license.

\section{Figures and Figure Legends}

a

b

Iteration 1:

- initial electroporation

condition

- individual sgRNAs

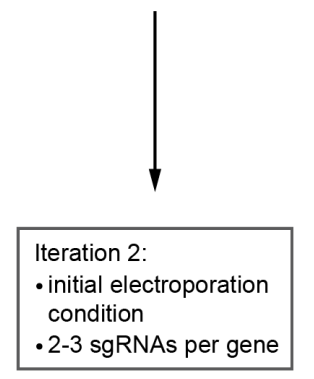

Iteration 3 (final):

- optimized electroporation condition

-2-3 sgRNAs per gene
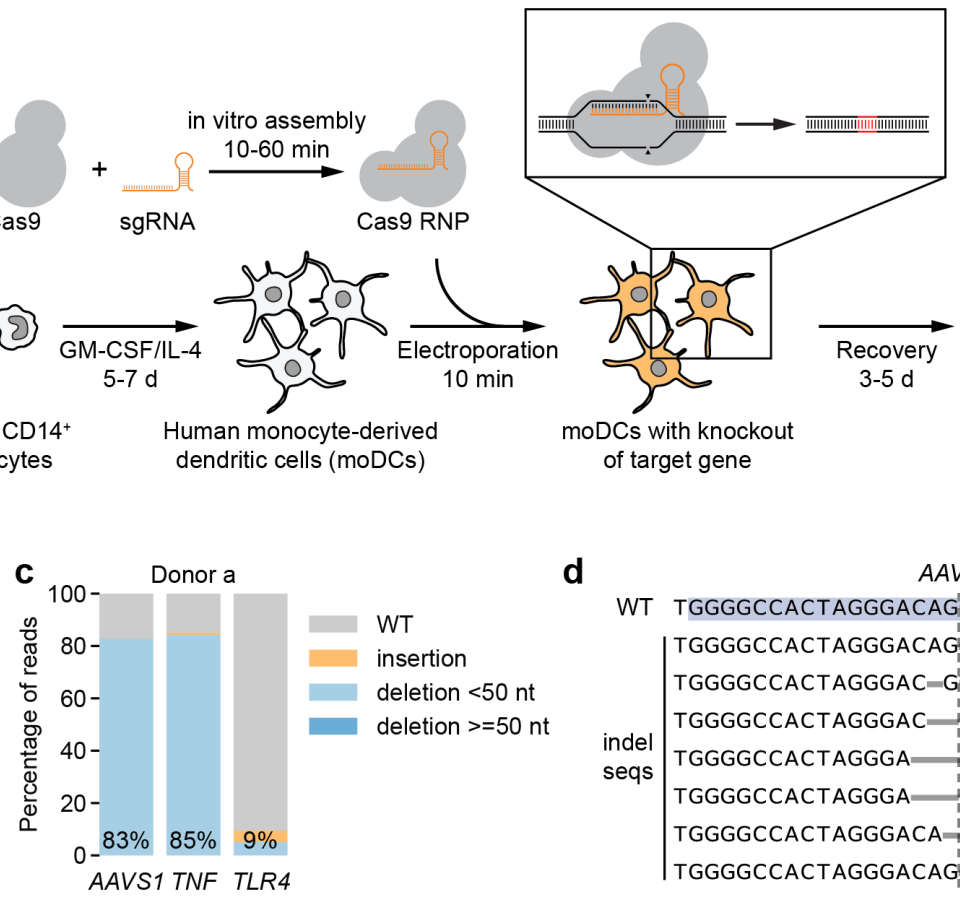

Figure 1

Genotyping by next-gen sequencing CGGACTACGTGGATGCACGG CGGACTACGTGGA-GCACGG CGGACTACGTGGAT-CACGG CGGACTACGTGGA--CACG CGGACTACGTGGACGCACGG CGGACTACGTGG - GCACGG

Phenotyping by profiling immune responses

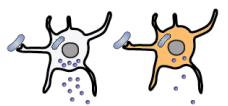

d

AAVS1

WT TGGgGCCACTAGgGaCAGGATTGGTGACAGA $17.08 \%$ TGGGGCCACTAGGGACAG-ATTGGTGACAGA $77.37 \%$ TGGGGCCACTAGGGAC-G'GATTGGTGACAGA $\quad 2.60 \%$ TGGGGCCACTAGGGAC - GATTGGTGACAGA $1.09 \%$

indel $\begin{array}{lll}\text { TGGGGCCACTAGGGA } & \text { TtGgtgaCAGA } & 0.28 \%\end{array}$ TGGGGCACTAGGGA GATTGGTGACAGA $0.19 \%$ TGGGGCCACTAGGGACA TGGTGACAGA $0.17 \%$ TGGGGCCACTAGGGACAG TGACAGA $0.15 \%$ $\overline{98.93 \%}$
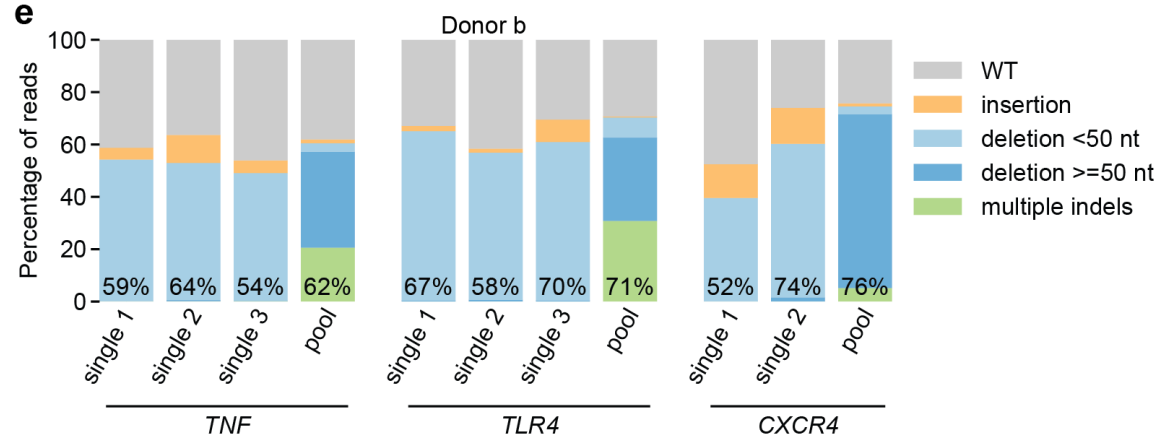

f

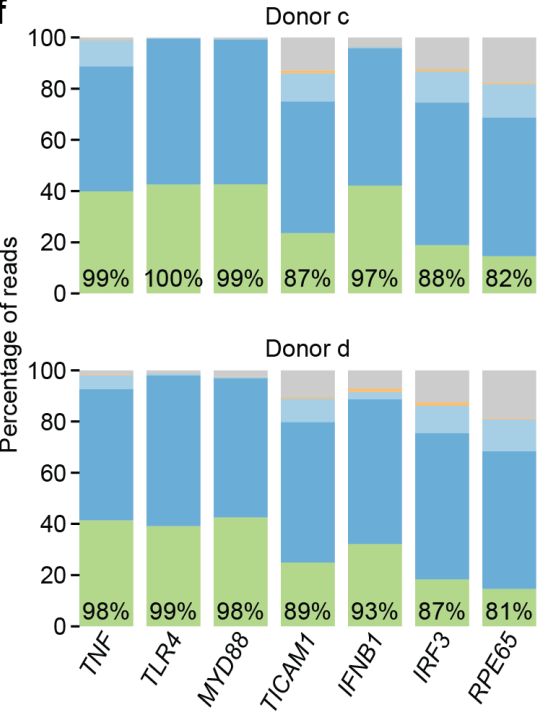

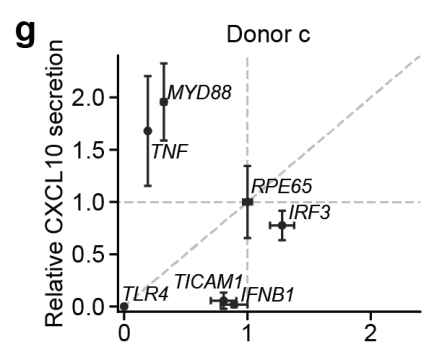

Donor d

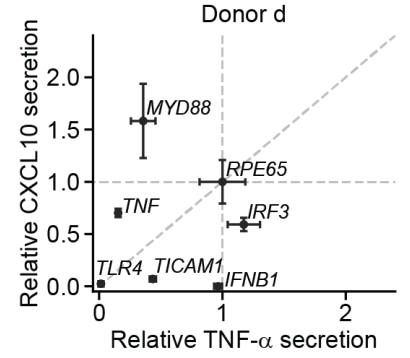


1077 Figure 1. CRISPR/Cas9-based strategy for gene knockout in human moDCs. (a) Schematic

1078 outline of the strategy. (b) Flow chart delineating optimization. (c) Percentage of genomic DNA

1079 reads assigned to different classes of outcomes after targeting each locus with Cas9 RNPs in

1080 condition P1, CB-150. Labeled values indicate total percentage of reads with a non-WT

1081 sequence. (d) Individual editing outcomes at the AAVS1 locus accounting for at least $0.15 \%$ of

1082 on-target reads. Horizontal gray bars denote deletions. Vertical dashed line denotes Cas9 cut site.

1083 (e) Outcome classification, as in (c), after targeting indicated loci with single or multi-sgRNA

1084 Cas9 RNPs. (f) Outcome classification, as in (c), after targeting 7 loci with multi-sgRNA Cas9

1085 RNPs in moDCs from two independent donors. (g) Production of TNF- $\alpha$ and CXCL10 by

1086 knockout moDCs challenged with $100 \mathrm{ng} / \mathrm{mL}$ E. coli O55 LPS, normalized to cell numbers and

1087 to cytokine production in moDCs with knockout of RPE65. Data represent mean and standard

1088 deviation of 2 independent treatments for both TNF- $\alpha$ and CXCL10 levels.

1089 See also Figure 1 - Figure Supplement 1, Figure 1 - Figure Supplement 2, and Figure 1 - Figure

1090 Supplement 3. 

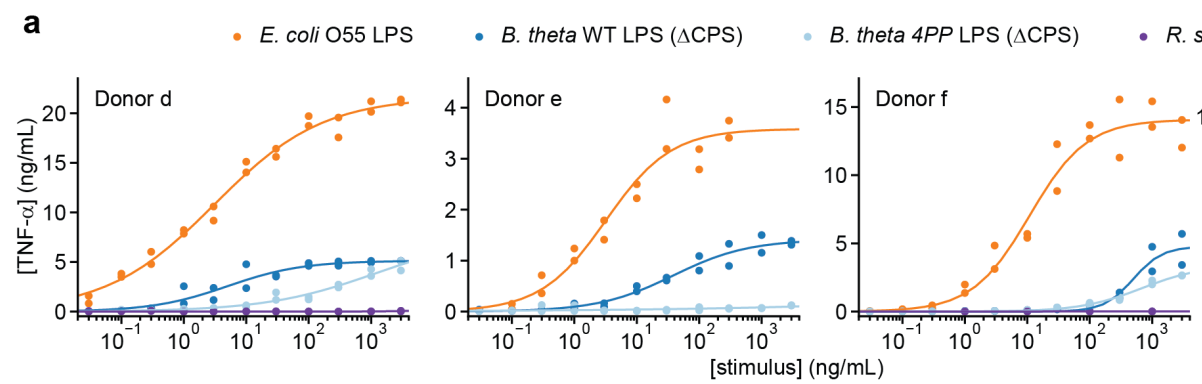

Figure 2

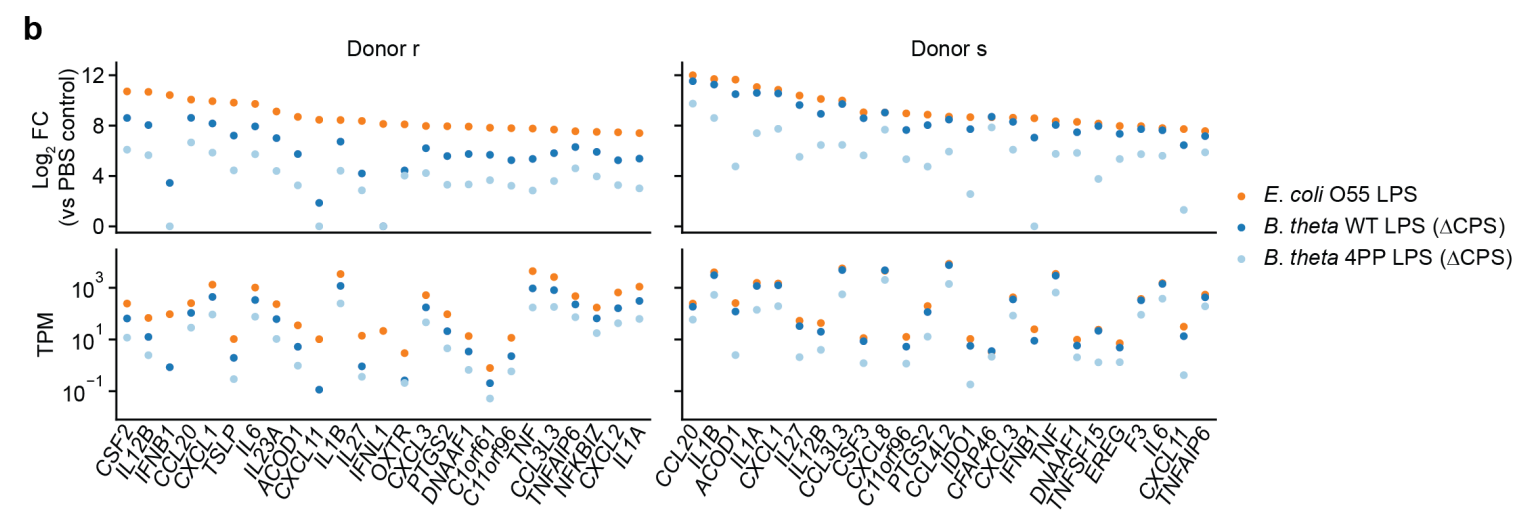

1094 Figure 2. Responses of human moDCs to LPSs are specific to bacterial species and human

1095 donor. (a) TNF- $\alpha$ secretion after stimulation of moDCs from 4 independent donors with titration 1096 series of the indicated LPSs. Cells from donor e were not treated with $R$. sphaeroides LPS. Each 1097 data point represents an independent treatment of 20,000 moDCs. Lines denote a Hill curve fit.

1098 (b) Expression levels of selected genes after stimulation of moDCs from two donors with 10

$1099 \mathrm{ng} / \mathrm{mL}$ E. coli O55 LPS, $100 \mathrm{ng} / \mathrm{mL}$ B. theta WT LPS, or $100 \mathrm{ng} / \mathrm{mL}$ B. theta 4PP LPS, as

1100 determined by RNA-seq. $\log _{2}$ fold-changes compared to PBS-treated control cells or transcript

1101 counts per million are shown for the 25 genes with the largest $\log _{2}$ fold-changes after treatment

1102 with E. coli O55 LPS. B. theta WT LPS elicits weaker upregulation of genes than E. coli O55

1103 LPS, with a more pronounced difference for genes downstream of TRIF such as IFNB1. B. theta

1104 4PP LPS elicits even weaker upregulation of genes. Data represent means obtained from three

1105 independent treatment replicates for each treatment and donor.

1106 See also Figure 2 - Figure Supplement 1 and Figure 2 - Figure Supplement 2. 
bioRxiv preprint doi: https://doi.org/10.1101/2020.12.22.423985; this version posted December 22, 2020. The copyright holder for this preprint (which was not certified by peer review) is the author/funder, who has granted bioRxiv a license to display the preprint in perpetuity. It is made available under aCC-BY 4.0 International license.

a
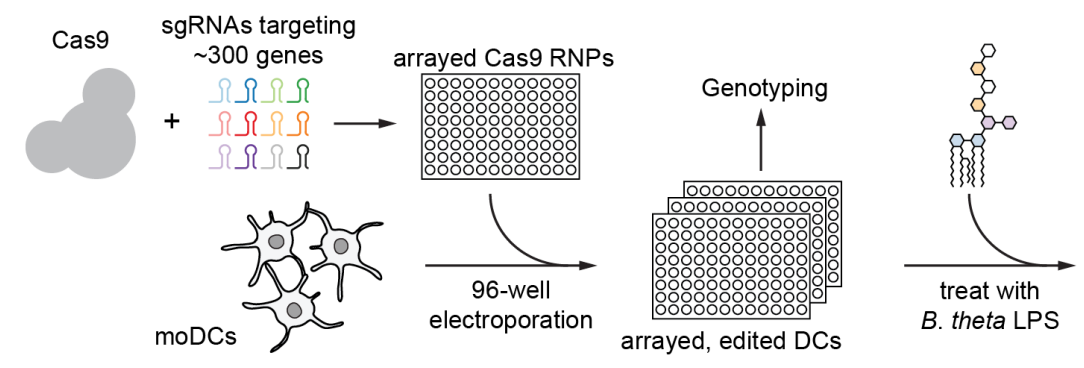

Figure 3
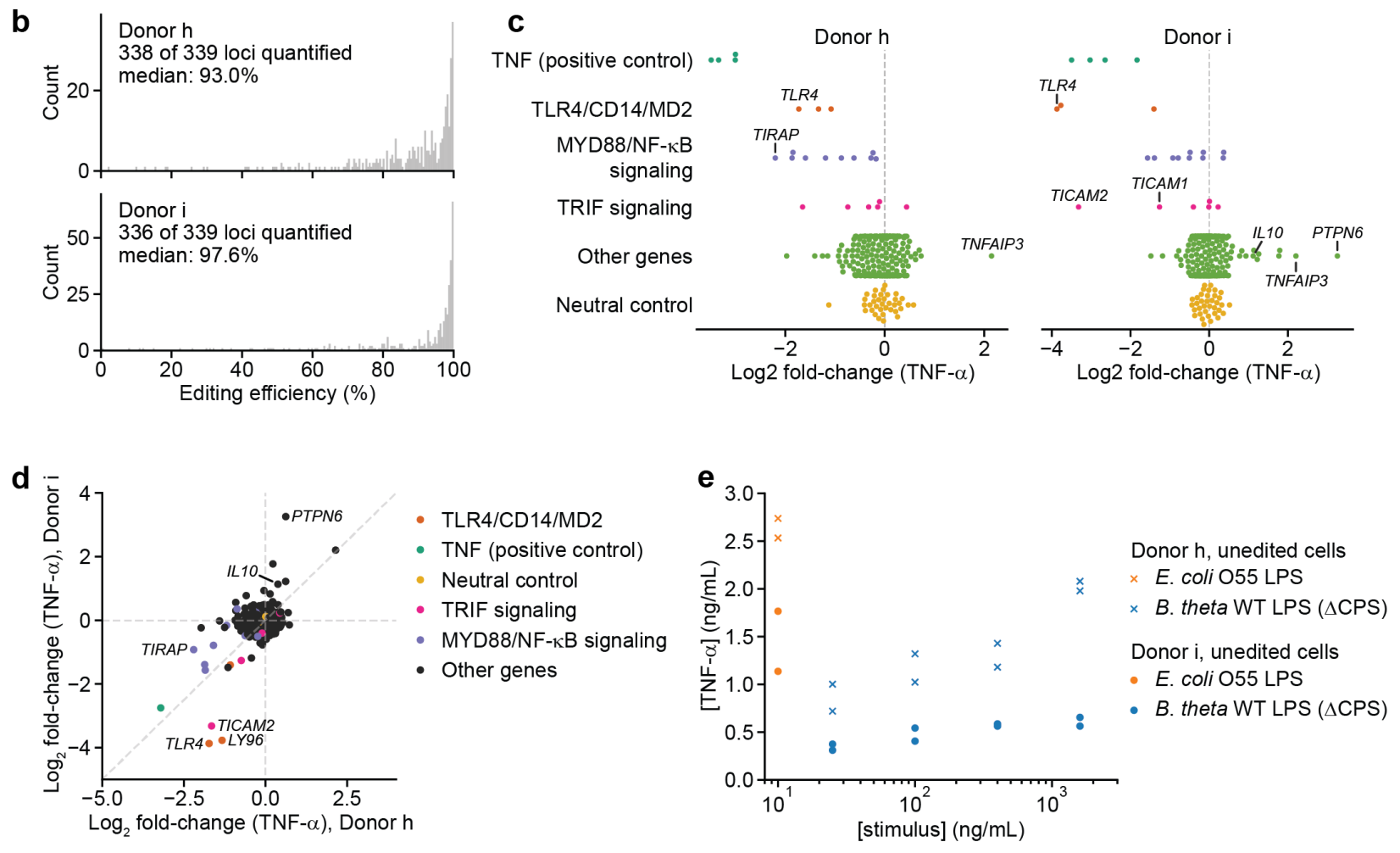

f
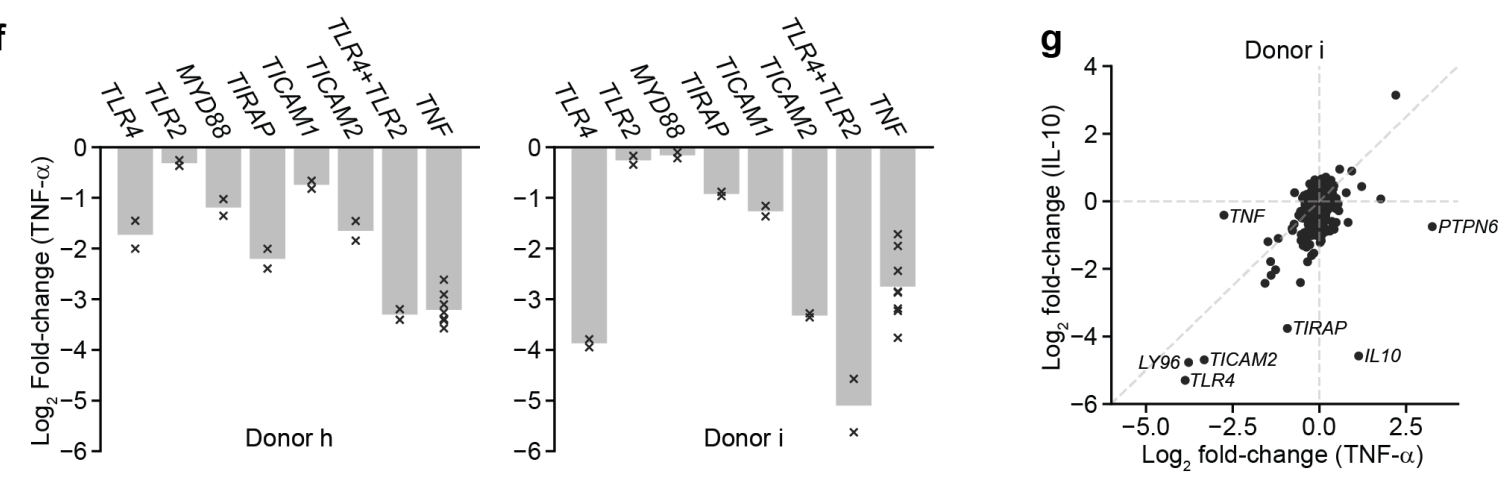

1108 Figure 3. An arrayed genetic screen reveals how moDCs recognize LPS from a human gut

1109 bacterium and mechanisms of inter-individual variation. (a) Schematic of genetic screen. (b) 
1110 Observed editing efficiencies for loci targeted in the screen. Efficiencies were not obtained for 1

1111 locus for donor $\mathrm{h}$ and 3 loci for donor i due to PCR failures; no locus failed for both donors. (c)

1112 TNF- $\alpha$ secretion of knockout moDC populations from two independent donors, displayed as $\log _{2}$

1113 fold-changes compared to neutral controls within each of the four 96-well plates and normalized

1114 to cell counts. Each data point represents the mean of two treatment replicates and two cell count

1115 replicates. (d) Comparison of TNF- $\alpha$ secretion from (c) for the two donors. (e) TNF- $\alpha$ secretion

1116 after stimulation of unedited moDCs (from no pulse/no RNP wells) from both donors with

1117 different concentrations of the indicated LPSs. Each data point represents an independent

1118 treatment. (f) TNF- $\alpha$ secretion for selected moDC knockout populations including moDCs with

1119 simultaneous knockout of TLR4 and TLR2. Data are shown as individual measurements $(\times)$ and

1120 mean of all treatment replicates (bars). (g) Comparison of TNF- $\alpha$ and IL-10 secretion from

1121 knockout moDC populations for moDCs derived from donor i. Each data point represents the

1122 mean of two treatment replicates and two cell count replicates for TNF- $\alpha$ secretion and data from

1123 a single treatment replicate and two cell count replicates for IL-10 secretion.

1124 See also Figure 3 - Figure Supplement 1, Figure 3 - Figure Supplement 2, Figure 3 - Figure

1125 Supplement 3, Figure 3 - Figure Supplement 4, Figure 3 - Figure Supplement 5, and Figure 3 -

1126 Figure Supplement 6. 
bioRxiv preprint doi: https://doi.org/10.1101/2020.12.22.423985; this version posted December 22, 2020. The copyright holder for this preprint (which was not certified by peer review) is the author/funder, who has granted bioRxiv a license to display the preprint in perpetuity. It is made available under aCC-BY 4.0 International license.

\section{Figure Supplements and Figure Supplement Legends}

a

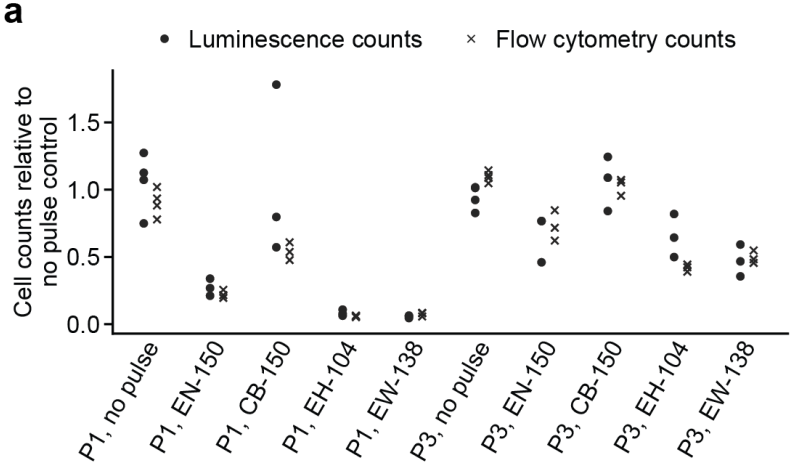

C

WT CACTTTGGAGTGATCGGCCCCCAGAGGGAGAGGTGAGTGC CACTTTGGAGTGATCGGCCCC-AGAGGGAAAGGTGAGTGC CACTTTGGAGTGATCGGCCC AGAGGGAAGAGGTGAGTGC CACTTTGGAGTGATCGGCCCC GAGGGAGAGGTGAGTGC CACTTTGGAGTGATCGGCC AGAGGGAGAGGTGAGTGC CACTTTGGAGTGAT—CAGAGGGAGAGGTGAGTGC

indel sequences CACTTTGGAGTGATCGG|CCCICIAGAGGGAAGAGGTGAGTGC CACTTTGGAGTGATCG CAGAGGGAAGAGGTGAGTGC CACTTTGGAGTGATCG CCCCAGAGGGAAGAGGTGAGTGC CACTTTGGAGTGAT:CAGAGGGAGAGGTGAGTGC CACTTTGGAGTGATCGGCCCC AGGGAAGAGGTGAGTGC CACTTTGGAG GGCCCCICAGAGGGAAGAGTGAGTGC CACTTTG CAGAGGGAGAGGTGAGTGC

d sgRNA (Synthego) TNF

crRNA:tracrRNA (IDT)

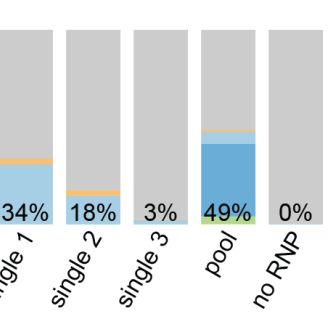

TLR4

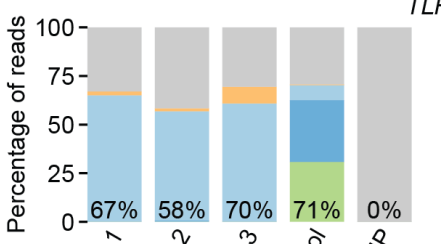

के के के के हें है

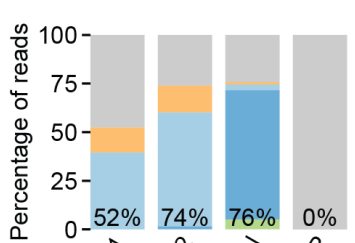

1131

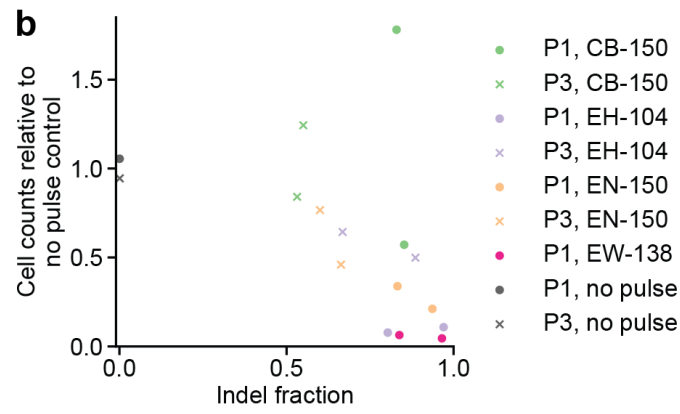
$14.78 \%$
$41.16 \%$
$25.91 \%$
$10.72 \%$
$2.50 \%$
$0.89 \%$
$0.70 \%$
$0.45 \%$
$0.34 \%$
$0.24 \%$
$0.21 \%$
$0.16 \%$
$\frac{0.15 \%}{98.34 \%}$

e
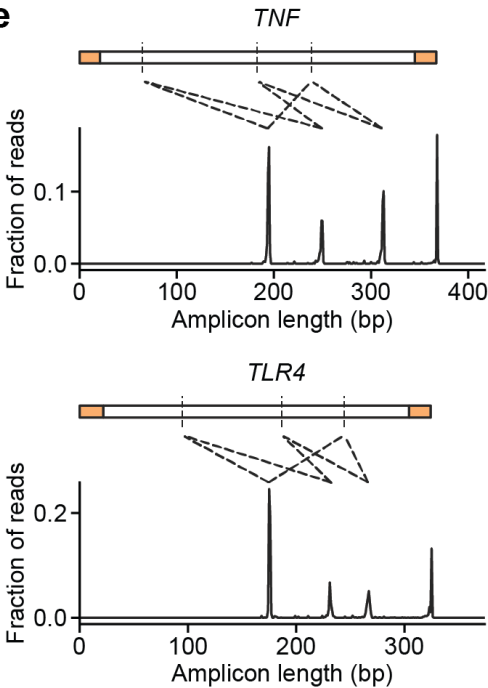

CXCR4

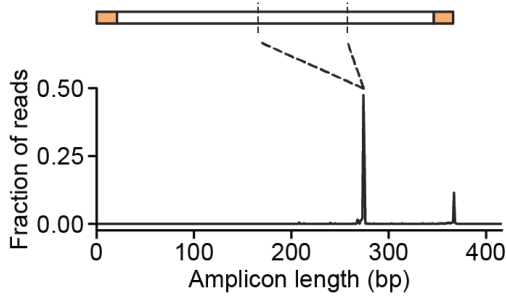


1132 Figure 1 - Figure Supplement 1. Additional characterization of initial conditions for moDC

1133 genome editing strategy. (a) Cell counts relative to no pulse control cells determined by two

1134 methods $3 \mathrm{~d}$ (flow cytometry) or $5 \mathrm{~d}$ (luminescence) after electroporation of moDCs in indicated

1135 conditions. (b) Comparison of indel fraction and cell counts measured in different

1136 electroporation conditions, identifying condition P1, CB-150 as a condition with low toxicity and

1137 high editing efficiency. (c) Individual editing outcomes at the TNF locus accounting for at least

$11380.15 \%$ of on-target reads after editing in condition P1, CB-150. Horizontal gray bars denote

1139 deletions. Vertical dashed line denotes Cas9 cut site. Superscripted bases denote insertions. Due

1140 to the presence of a C homopolymer at the cut site, the location of some short deletions and

1141 insertions cannot be unambiguously inferred; the most likely location is denoted. (d) Percentage

1142 of genomic DNA reads assigned to different classes of outcomes after targeting each locus with

1143 single or multi-sgRNA Cas9 RNPs in condition P1, CB-150. Cas9 RNPs were formed either with

1144 sgRNAs (left) or crRNA:tracrRNA complexes (right). Labeled values indicate total percentage

1145 of reads with a non-WT sequence. (e) Distribution of amplicon lengths for moDCs

1146 electroporated with multi-sgRNA Cas9 RNPs (in the sgRNA format). Observed amplicons

1147 correspond to deletions of regions between sgRNA cut sites, as indicated by the diagrams.

1148 Amplicons are depicted with the primer binding sequences in orange and sgRNA cut sites as

1149 vertical dashed lines. 
bioRxiv preprint doi: https://doi.org/10.1101/2020.12.22.423985; this version posted December 22, 2020. The copyright holder for this preprint (which was not certified by peer review) is the author/funder, who has granted bioRxiv a license to display the preprint in perpetuity. It is made available under aCC-BY 4.0 International license.

\section{a}
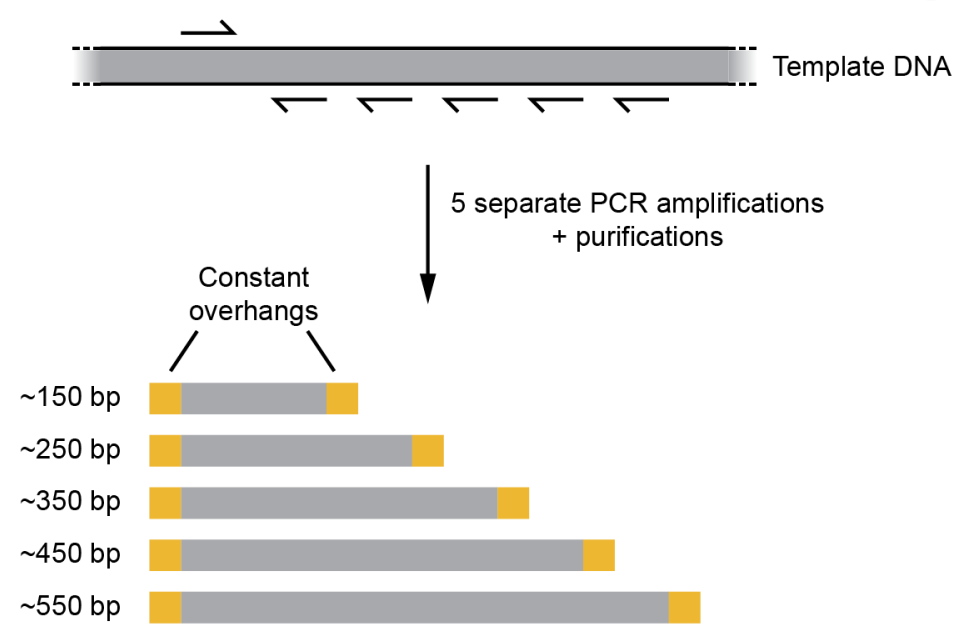

Mix at 11 ratios

Figure 1 - Figure Supplement 2

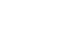
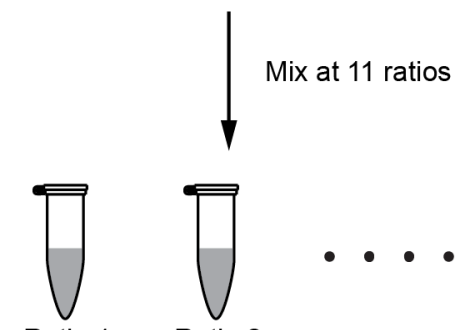

Ratio 1

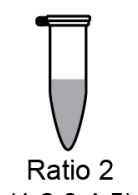

$(1: 1: 1: 1: 1) \quad(1: 2: 3: 4: 5)$
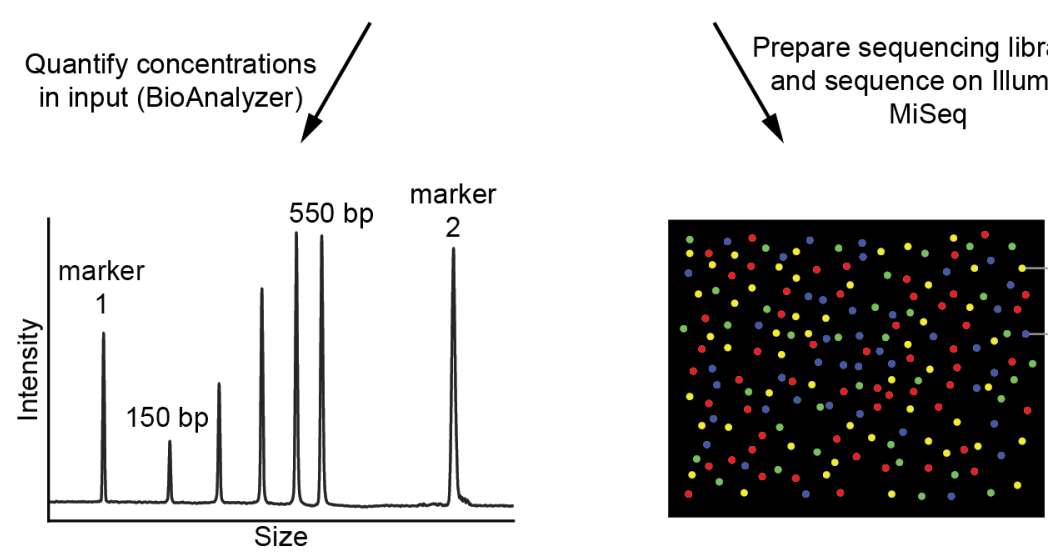

$150 \mathrm{bp}$

-550 bp

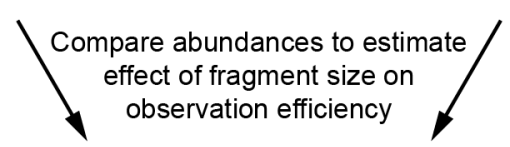

b

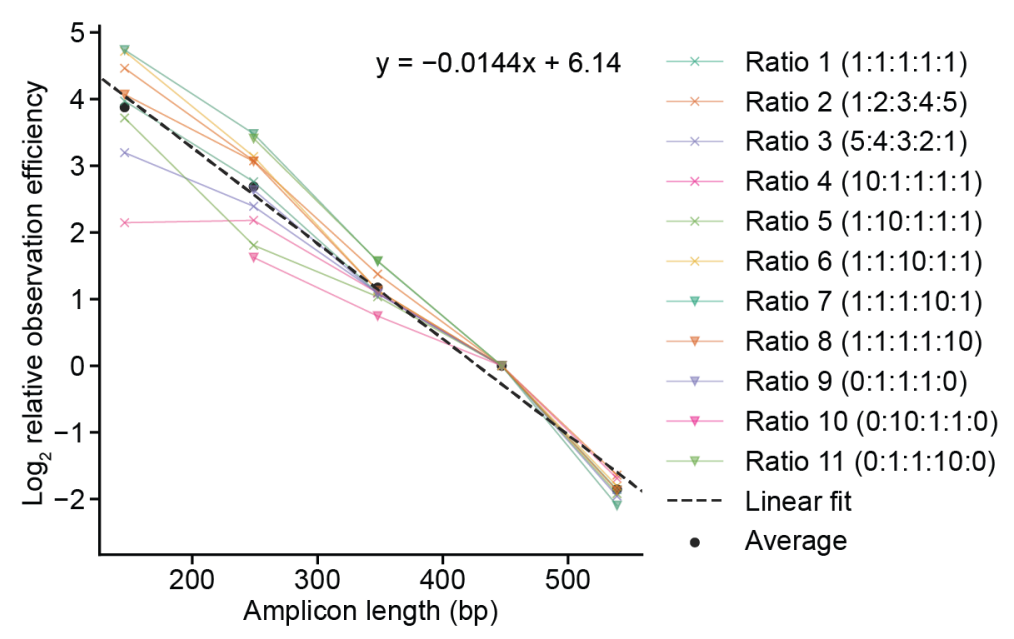


1153 Figure 1 - Figure Supplement 2. Strategy to measure effect of amplicon size on observation

1154 (amplification + sequencing) efficiency. (a) Schematic outline of strategy. See methods for

1155 details. (b) Comparison of calculated observation efficiencies and fragment size for each

1156 fragment with each of the 11 reaction ratios. Dotted line represents fit derived from a linear

1157 regression, with coefficients indicated.

1158

1159 
Figure 1 - Figure Supplement 3

a

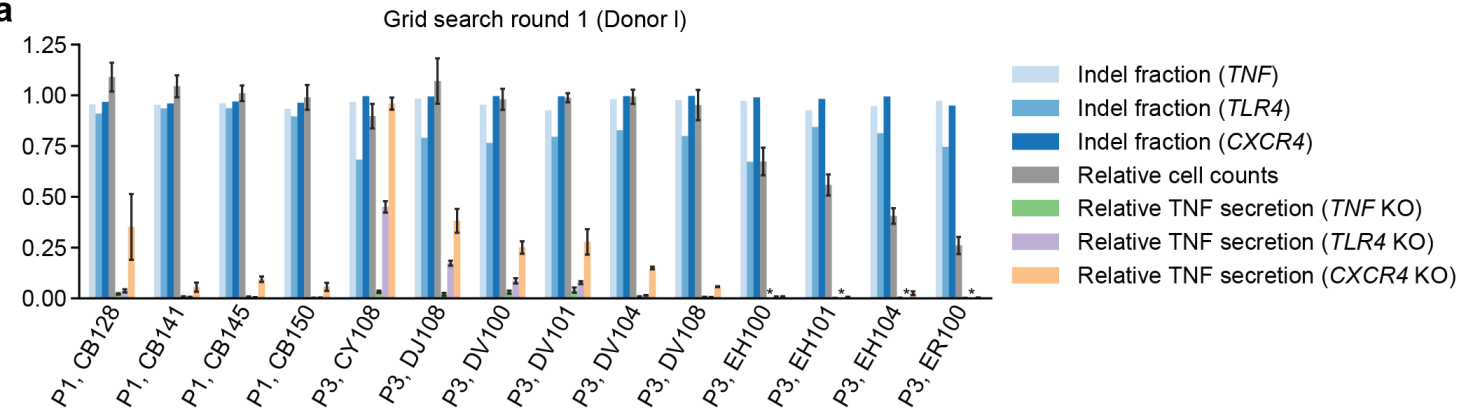

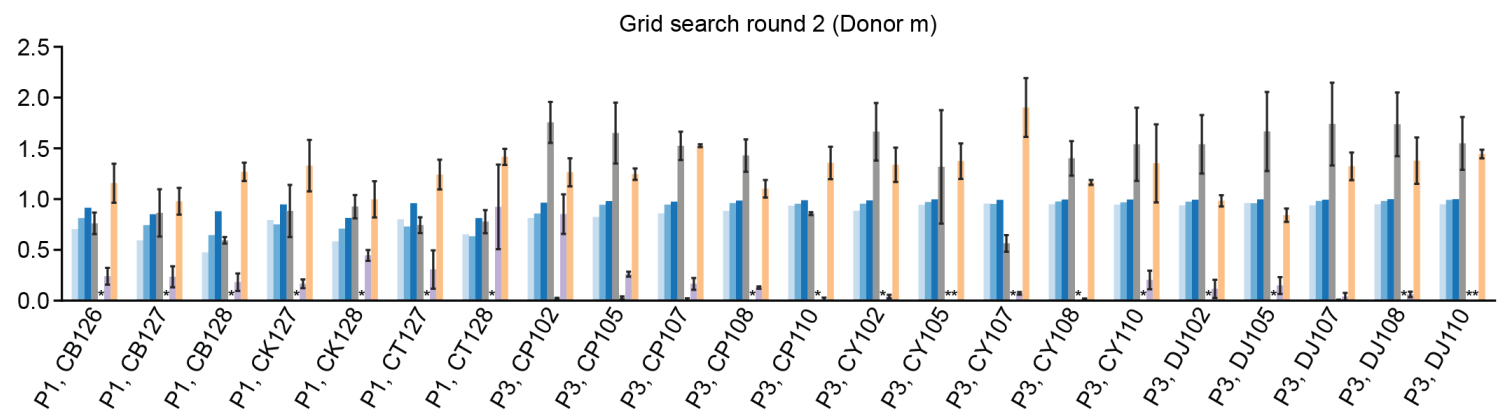

b
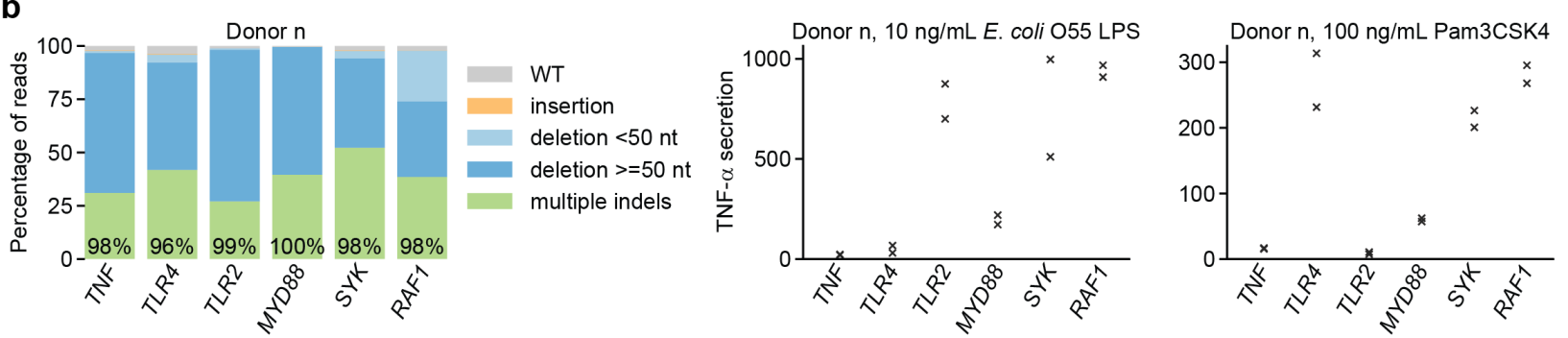

1160
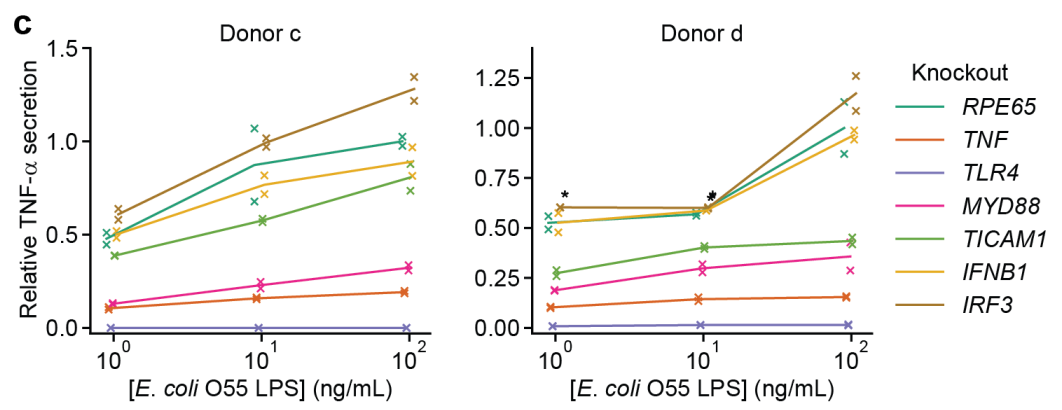

1161 Figure 1 - Figure Supplement 3. Identification of an optimal electroporation condition for

1162 moDC genome editing. (a) Results from two successive grid searches of electroporation

1163 conditions. Ideal conditions have high indel fraction at all loci, high cell counts, low TNF- $\alpha$

1164 secretion in response to $10 \mathrm{ng} / \mathrm{mL}$ E. coli LPS with knockout of TNF and TLR4, and high TNF- $\alpha$ 
1165 secretion with knockout of $C X C R 4$. TNF- $\alpha$ secretion was normalized to cell counts. Indel

1166 fractions represent data from a single measurement. Cell counts represent mean count \pm standard

1167 deviation from two independent wells. TNF- $\alpha$ secretion values represent mean \pm standard

1168 deviation of two independent treatments, normalized to cell counts. $(*)$ indicates signal below

1169 detection limit. (b) Left: Percentage of genomic DNA reads assigned to different classes of

1170 outcomes after targeting each locus with multi-sgRNA Cas9 RNPs. Labeled values indicate total

1171 percentage of reads with a non-WT sequence. Right: Production of TNF- $\alpha$ by knockout moDCs

1172 challenged with $10 \mathrm{ng} / \mathrm{mL}$ E. coli O55 LPS or $100 \mathrm{ng} / \mathrm{mL}$ Pam3CSK4, normalized to cell counts.

1173 Data represent two independent treatments, normalized to cell count obtained from replicate

1174 measurements. (c) Production of TNF- $\alpha$ by knockout moDCs challenged with indicated

1175 concentrations of E. coli O55 LPS, normalized to cell counts and to TNF- $\alpha$ production by

1176 moDCs with knockout of RPE65 and treated with the highest concentration of E. coli O55 LPS.

1177 All samples were treated with identical LPS concentrations, but x-values are slightly offset by

1178 sample for clarity. Data represent two independent treatments, normalized to cell count obtained

1179 from replicate measurements. (*) indicates sample saturated during measurement of TNF- $\alpha$

1180 secretion. Data from treatment with $100 \mathrm{ng} / \mathrm{mL}$ E. coli O55 LPS are also plotted in Figure $1 \mathrm{~g}$. 
a

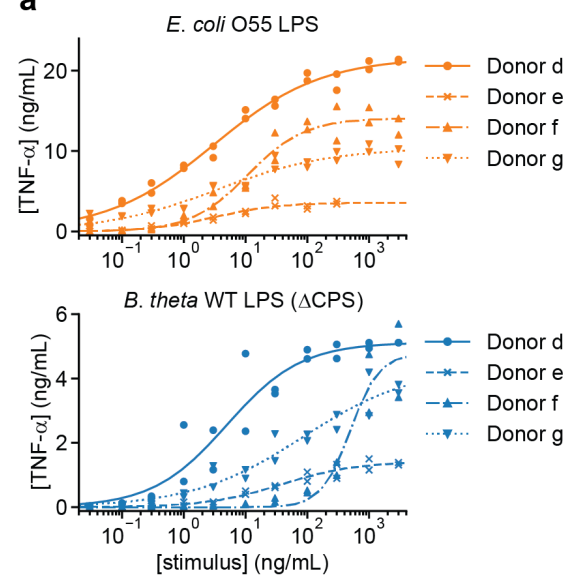

Figure 2 - Figure Supplement 1

b

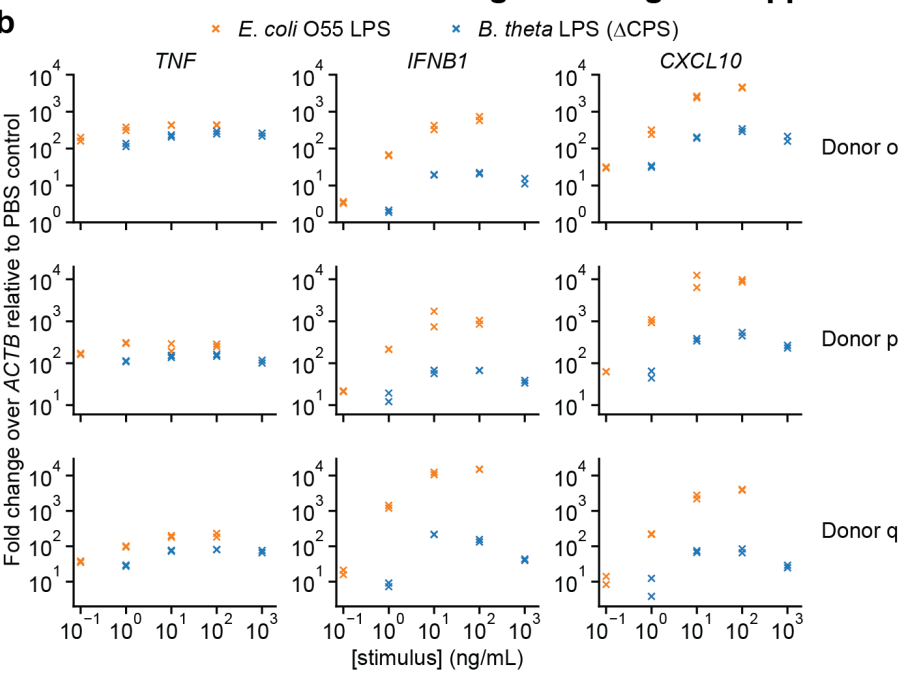

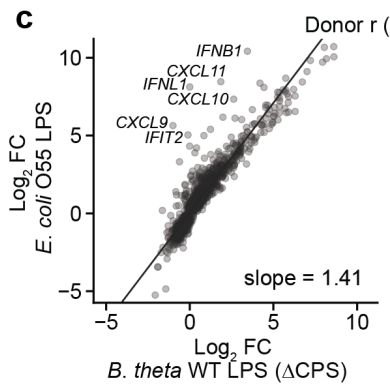

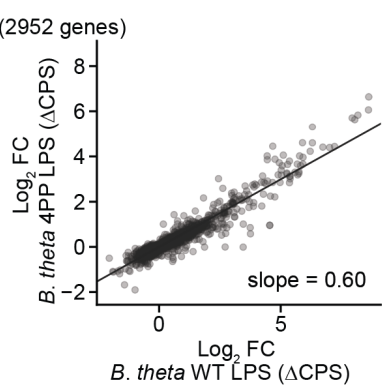

$\log _{2}$ FC
B. theta WT LPS ( $\triangle \mathrm{CPS})$

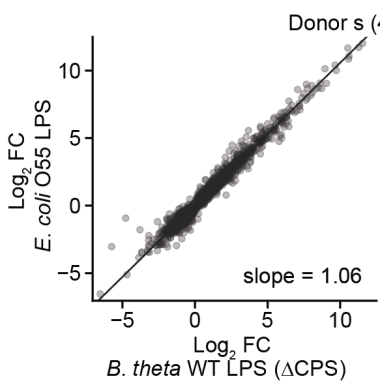

B. theta WT LPS ( $\triangle \mathrm{CPS})$

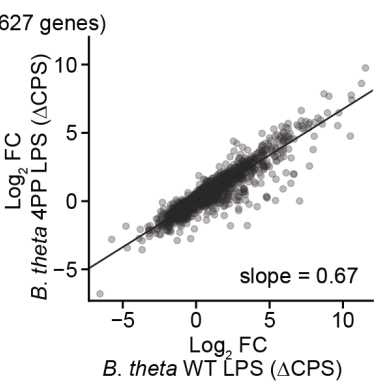

1184 Figure 2 - Figure Supplement 1. B. theta LPS and E. coli LPS elicit different responses from

1185 moDCs with responses that vary by donor. (a) Responses to individual LPSs from Figure 2a

1186 plotted by donor to highlight differences in response magnitude and $\mathrm{EC}_{50}$. (b) Fold-changes in

1187 gene expression for TNF (activated by MYD88 and TRIF), IFNB1, and CXCL10 (both activated

1188 only by TRIF) upon treatment with $B$. theta LPS or E. coli LPS for moDCs derived from three

1189 independent donors, measured by qPCR as $2^{-\Delta \Delta \mathrm{Cp}}$, calculated as the difference in $\mathrm{C}_{\mathrm{p}}$ of the target

1190 gene compared to $A C T B(\triangle \mathrm{Cp})$ and normalized to the same quantity for PBS-treated cells

$1191(\Delta \Delta \mathrm{Cp})$. Data represent two independent treatments and RNA extractions. Each data point

1192 represents the mean of three technical replicates. (c) Comparison of $\log _{2}$ fold-changes from

1193 RNA-seq data for all genes differentially expressed with $p<0.01$ with any treatment, as

1194 determined by DeSeq2 (Love et al., 2014). B. theta WT LPS elicits weaker upregulation of genes 
1195 than E. coli LPS, although the difference is weaker for donor s. B. theta 4PP LPS elicits weaker

1196 upregulation of genes than $B$. theta WT LPS. Data represent means obtained from three

1197 independent treatment replicates for each treatment and donor. Lines denote fit from a linear

1198 regression, with slope indicated. 
bioRxiv preprint doi: https://doi.org/10.1101/2020.12.22.423985; this version posted December 22, 2020. The copyright holder for this preprint (which was not certified by peer review) is the author/funder, who has granted bioRxiv a license to display the preprint in perpetuity. It is made available under aCC-BY 4.0 International license.
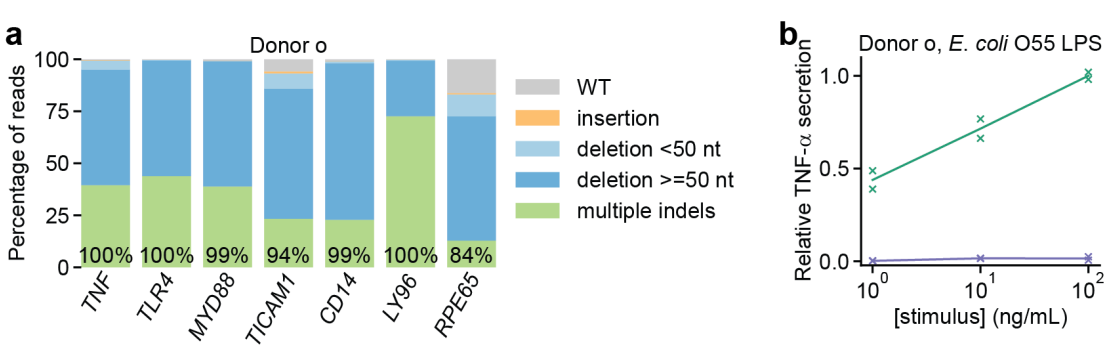

Figure 2 - Figure Supplement 2
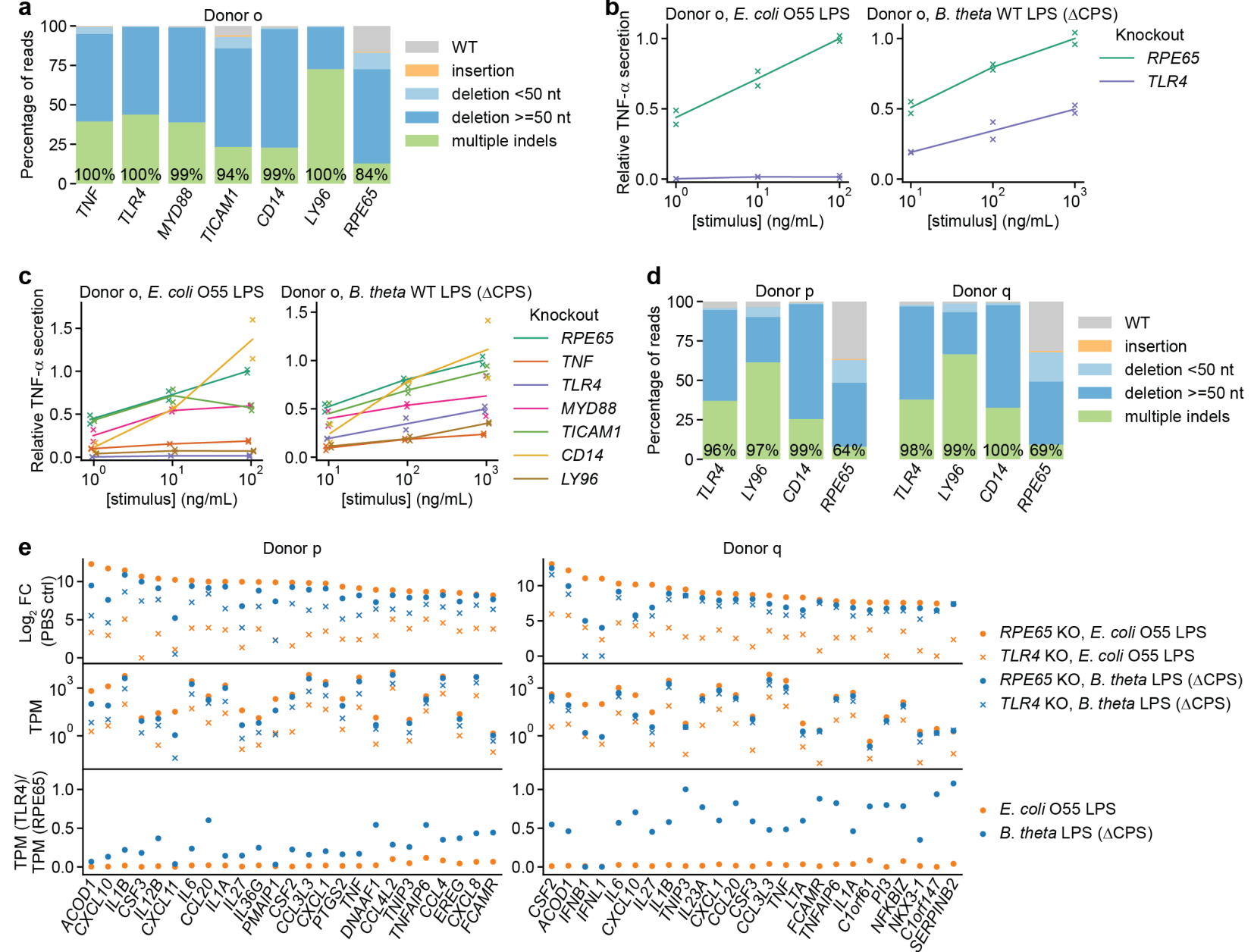

f
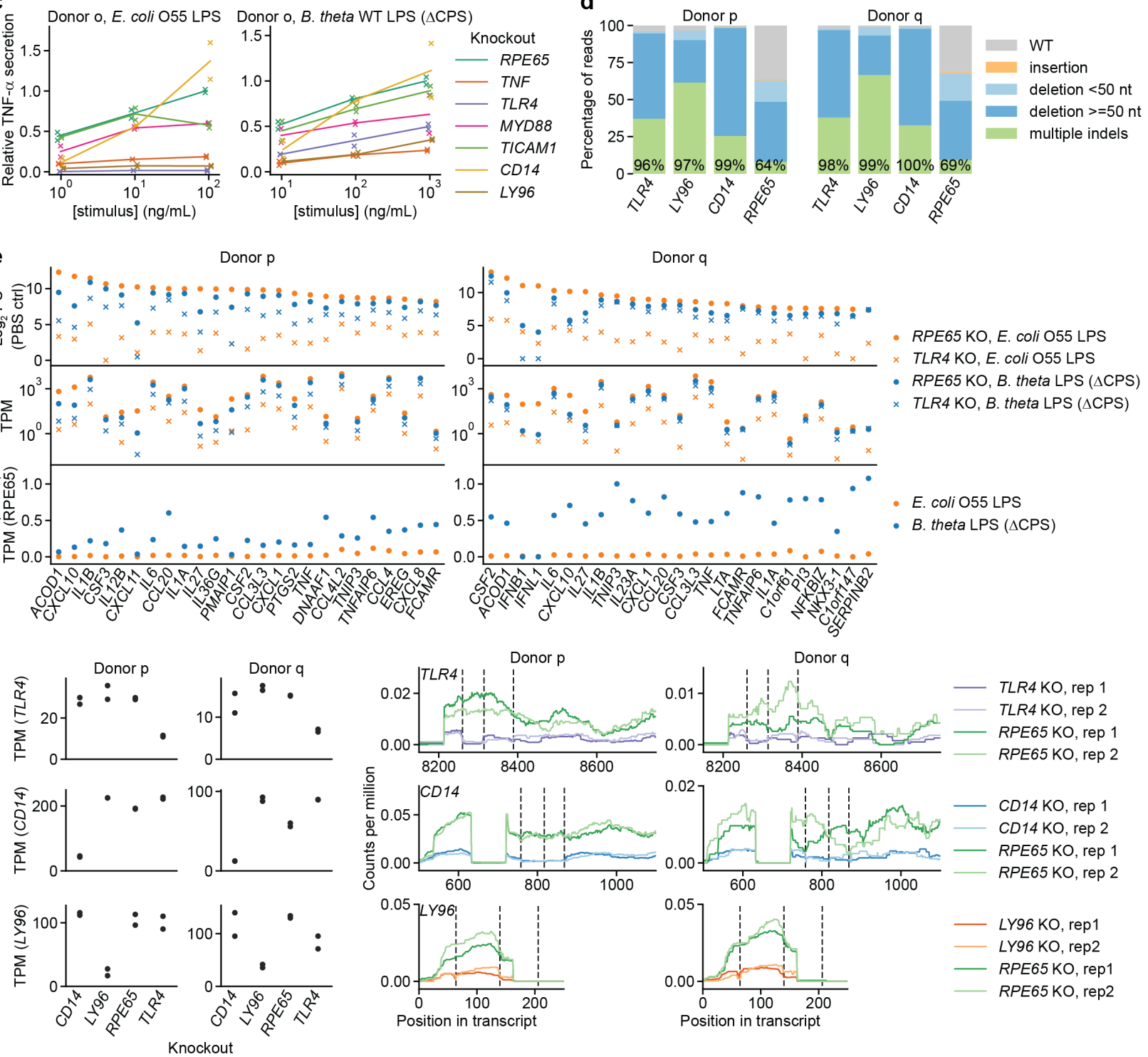

1200 Figure 2 - Figure Supplement 2. TLR4 knockout does not completely eliminate the response to

1201 B. theta LPS. (a) Percentage of genomic DNA reads assigned to different classes of outcomes

1202 after targeting each locus with multi-sgRNA Cas9 RNPs. Labeled values indicate total 
1203 percentage of reads with a non-WT sequence. (b) Production of TNF- $\alpha$ by moDCs with

1204 knockout of TLR4 or RPE65 challenged with indicated concentrations of E. coli O55 LPS or $B$.

1205 theta LPS, normalized to cell counts and to TNF- $\alpha$ production by moDCs with knockout of

1206 RPE65 and treated with the highest concentration of the respective LPS. Data represent two

1207 independent treatments, normalized to cell count obtained from replicate measurements. (c)

1208 Production of TNF- $\alpha$ by knockout moDCs, as in (b), with data from additional knockouts also

1209 shown. All samples were treated with identical LPS concentrations, but x-values are slightly

1210 offset by sample for clarity. (d) Percentage of genomic DNA reads assigned to different classes

1211 of outcomes after targeting each locus with multi-sgRNA Cas9 RNPs. Labeled values indicate

1212 total percentage of reads with a non-WT sequence. (e) Expression levels of selected genes after

1213 stimulation of knockout moDCs from two donors with $3 \mathrm{ng} / \mathrm{mL}$ E. coli O55 LPS and $100 \mathrm{ng} / \mathrm{mL}$

1214 B. theta WT LPS (donor p) or $10 \mathrm{ng} / \mathrm{mL}$ E. coli O55 LPS and $30 \mathrm{ng} / \mathrm{mL}$ B. theta WT LPS (donor

1215 q). Treatment concentrations were chosen based on similar transcriptional upregulation observed

1216 by qPCR (Figure 2 - Figure Supplement 1 b). $\log _{2}$ fold-changes compared to control cells (PBS-

1217 treated, knockout of RPE65), transcript counts per million (TPM), or the ratio of TPM in moDCs

1218 with knockout of TLR4 and moDCs with knockout of RPE65 are shown for the 25 genes with the

1219 largest summed $\log _{2}$ fold-changes after treatment with E. coli O55 LPS and B. theta WT LPS in

1220 moDCs with knockout of RPE65. MoDCs with TLR4 knockout exhibit almost no transcriptional

1221 changes in response to $E$. coli LPS but upregulate many genes in response to $B$. theta WT LPS,

1222 with the exception of genes downstream of TRIF, such as IFNB1. Data represent means obtained

1223 from two independent treatment replicates for each treatment and donor. (f) Assessment of

1224 transcript abundance for targeted genes in knockout moDC populations, revealing reduced

1225 transcript counts for targeted genes and further reduced mRNA coverage around sgRNA cut 
1226 sites. Left: Transcript counts (transcripts per million) for targeted genes in different PBS-treated

1227 (control) knockout moDC populations. Data represent measurements from independent

1228 replicates. Right: Transcript read coverage for targeted genes in different PBS-treated (control)

1229 knockout moDC populations. sgRNA cut sites are indicated by vertical dashed lines. Only exons

1230 with the sgRNA cut sites are shown.

1231

1232 
bioRxiv preprint doi: https://doi.org/10.1101/2020.12.22.423985; this version posted December 22, 2020. The copyright holder for this preprint (which was not certified by peer review) is the author/funder, who has granted bioRxiv a license to display the preprint in perpetuity. It is made available under aCC-BY 4.0 International license.

Figure 3 - Figure Supplement 1
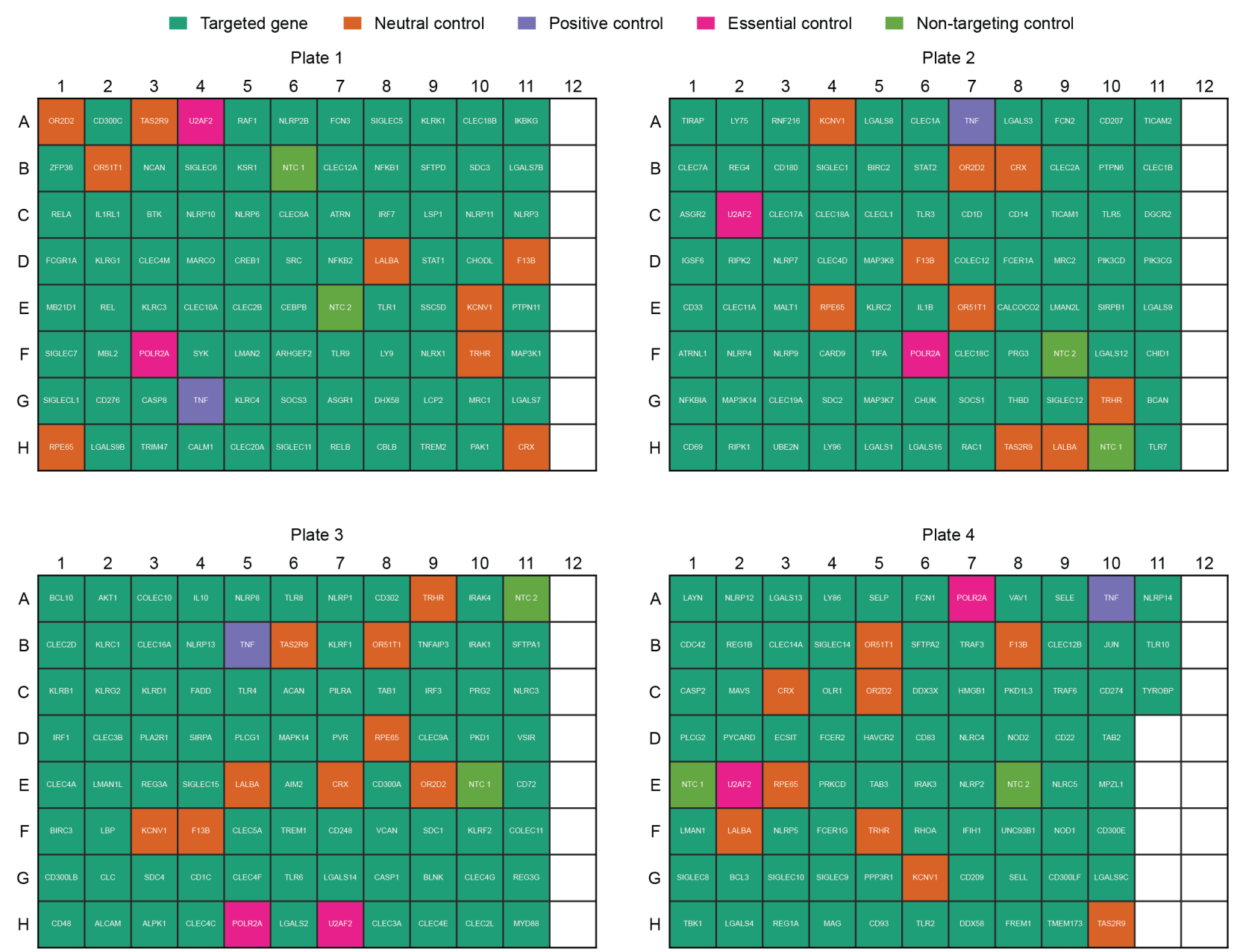

1234 Figure 3 - Figure Supplement 1. Layout of sgRNAs in the arrayed genetic screen. Non-

1235 targeting controls and sgRNAs targeting neutral controls, essential controls, and TNF (positive

1236 control) were included on each plate in randomized positions. 
bioRxiv preprint doi: https://doi.org/10.1101/2020.12.22.423985; this version posted December 22, 2020. The copyright holder for this preprint (which was not certified by peer review) is the author/funder, who has granted bioRxiv a license to display the preprint in perpetuity. It is made available under aCC-BY 4.0 International license.
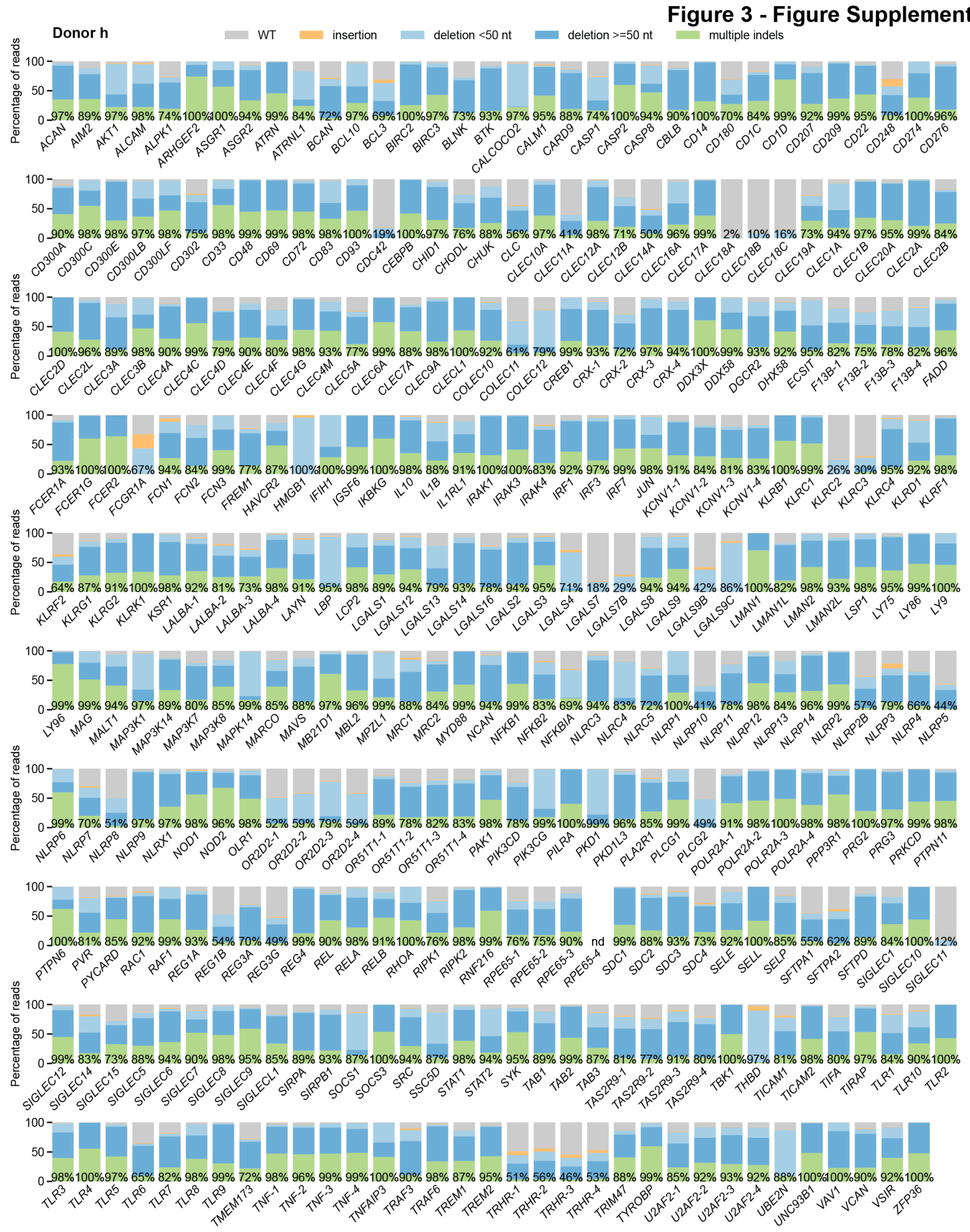

1240 Figure 3 - Figure Supplement 2. Percentage of genomic DNA reads assigned to different

1241 classes of outcomes after targeting each locus in the arrayed genetic screen (donor h). Labeled 
1242 values indicate total percentage of on-target reads with a non-WT sequence. For loci with

1243 percentages labeled as “nd”, sequencing library preparation failed and editing efficiency was not

1244 quantified. For genes targeted on each plate, editing efficiency was estimated for each plate

1245 individually. Loci are denoted as locus-\# with \# indicating the plate number. 
bioRxiv preprint doi: https://doi.org/10.1101/2020.12.22.423985; this version posted December 22, 2020. The copyright holder for this preprint (which was not certified by peer review) is the author/funder, who has granted bioRxiv a license to display the preprint in perpetuity. It is made available under aCC-BY 4.0 International license.

Figure 3 - Figure Supplement 3
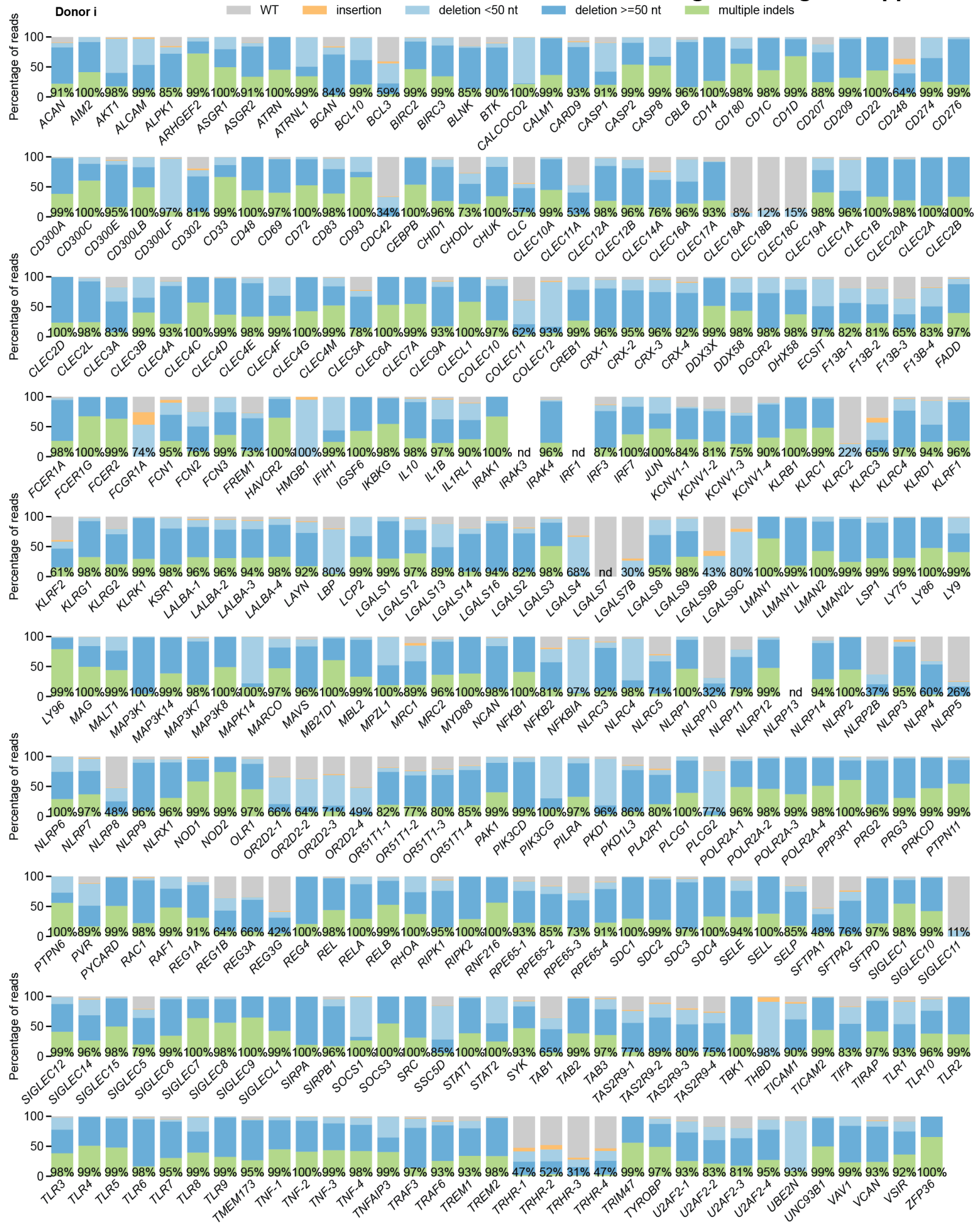

1249 Figure 3 - Figure Supplement 3. Percentage of genomic DNA reads assigned to different

1250 classes of outcomes after targeting each locus in the arrayed genetic screen (donor i). Labeled 
1251 values indicate total percentage of on-target reads with a non-WT sequence. For loci with

1252 percentages labeled as “nd”, sequencing library preparation failed and editing efficiency was not

1253 quantified. For genes targeted on each plate, editing efficiency was estimated for each plate

1254 individually. Loci are denoted as locus-\# with \# indicating the plate number.

1255

1256 
a

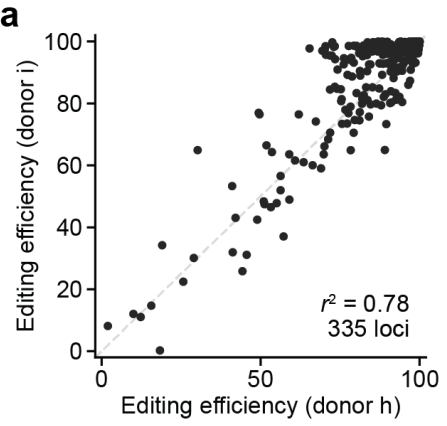

C

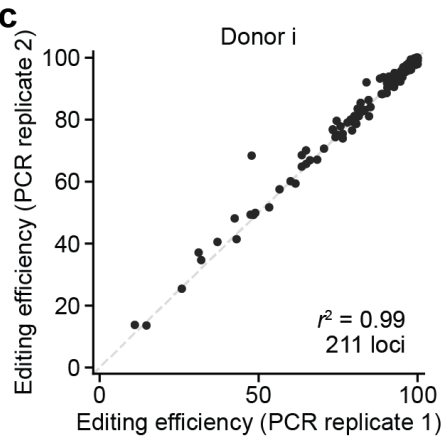

Figure 3 - Figure Supplement 4
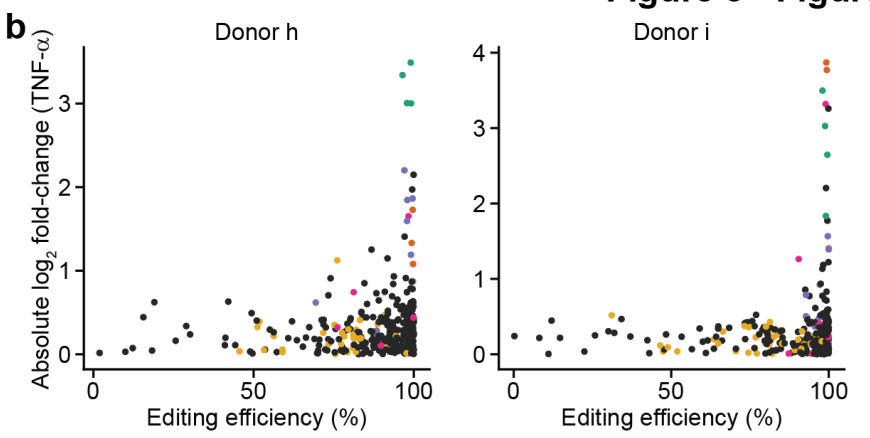

- TNF (positive control)

- TLR4/CD14/MD2

- MYD88/NF-kB signaling

- TRIF signaling

- Neutral control

- Other genes

1258 Figure 3 - Figure Supplement 4. Further analysis of editing outcomes from genetic screens. (a)

1259 Comparison of editing efficiencies observed in two independent donors for 335 loci. $r^{2}$ denotes

1260 squared Pearson correlation. (b) Comparison of screen phenotype (absolute value of $\log _{2}$ fold-

1261 change in TNF- $\alpha$ secretion after stimulation with $B$. theta LPS, mean of two treatment replicates)

1262 and editing efficiency, suggesting that high editing efficiency is required for a strong screen

1263 phenotype. (c) Comparison of editing efficiencies observed for two sequencing libraries prepared

1264 and sequenced independently, suggesting that the sequencing library preparation protocol and

1265 correction for size bias result in reproducible editing efficiencies. The second replicate was only

1266 performed for 211 loci. (d) Percentage of genomic DNA reads assigned to different classes of

1267 outcomes after targeting indicated loci with Cas9 RNPs. Labeled values indicate total percentage

1268 of reads with a non-WT sequence. For donor i, editing efficiency at the TLR4 locus was not

1269 determined for the cell population simultaneously electroporated with Cas9 RNPs targeting

$1270 \quad$ TLR4 and TLR2. 
bioRxiv preprint doi: https://doi.org/10.1101/2020.12.22.423985; this version posted December 22, 2020. The copyright holder for this preprint (which was not certified by peer review) is the author/funder, who has granted bioRxiv a license to display the preprint in perpetuity. It is made available under aCC-BY 4.0 International license.

Figure 3 - Figure Supplement 5

a
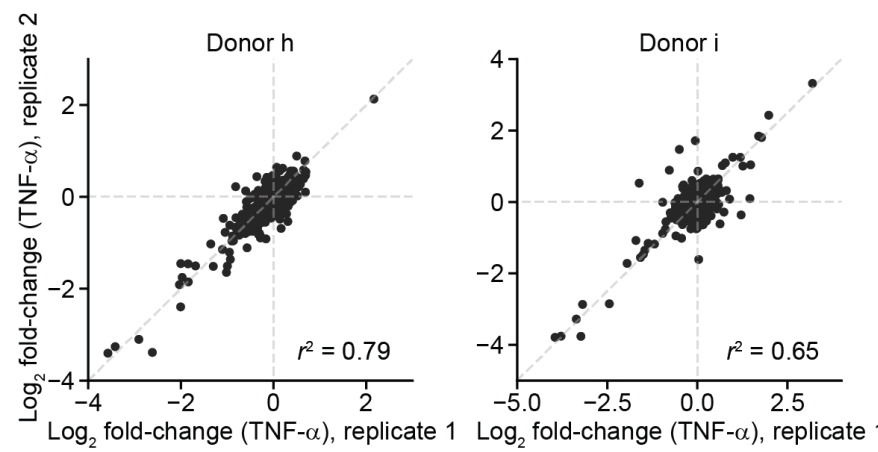

b

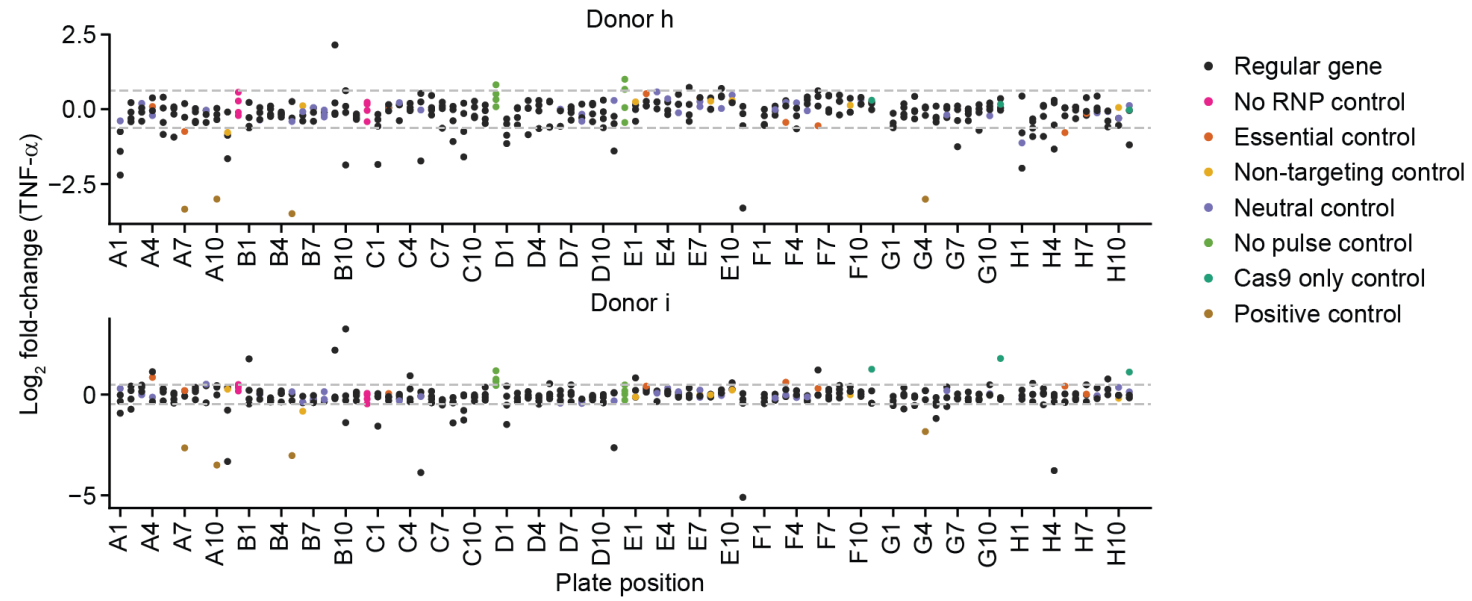

C

- TLR4/CD14/MD2 - TNF (positive control) - Neutral control - TRIF signaling - MYD88/NFkB signaling • Other genes
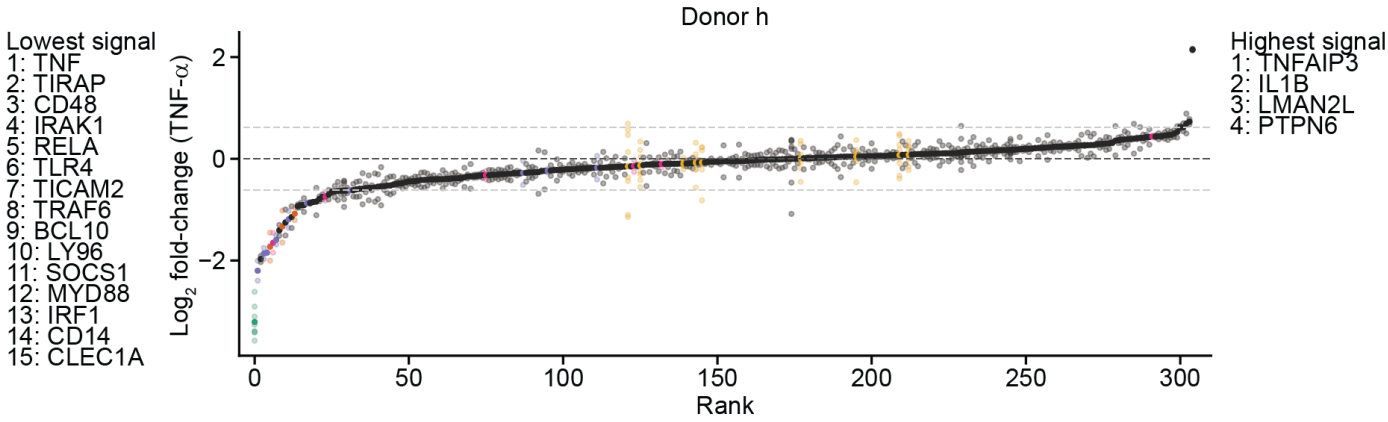

1271
Donor i
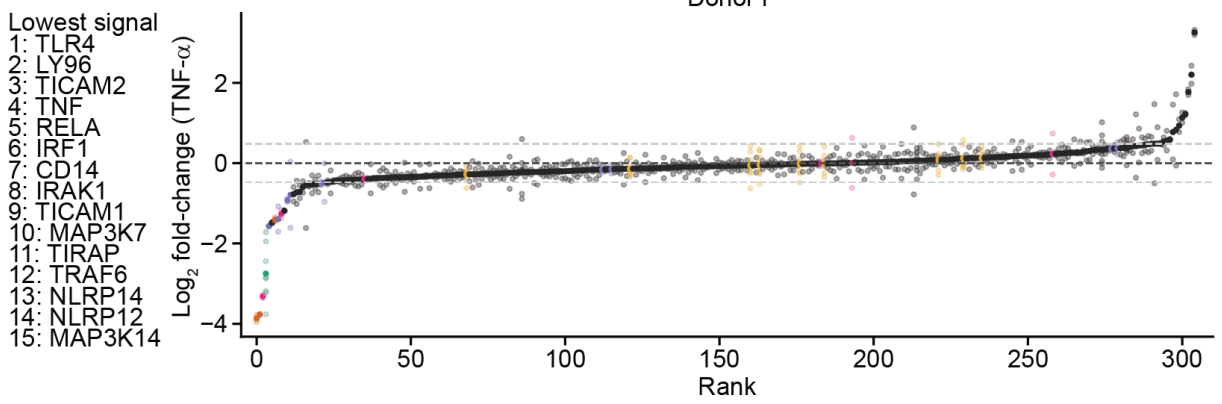

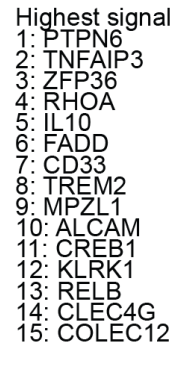


1272 Figure 3 - Figure Supplement 5. Screen phenotypes. (a) Comparison of phenotypes derived

1273 from replicate treatments for each donor. Each data point represents data from a single treatment

1274 replicate and two cell count replicates. $r^{2}$ denotes squared Pearson correlation. (b) Screen

1275 phenotypes ( $\log _{2}$ fold-changes in TNF- $\alpha$ secretion relative to neutral controls) stratified by plate

1276 position. Each data point represents the average of two replicate treatments and two cell count

1277 replicates. Horizontal dashed lines denote 2 standard deviations of the phenotypes from all

1278 neutral gene controls. (c) Screen phenotypes (log2 fold-changes in TNF- $\alpha$ secretion) ordered by

1279 magnitude. Solid points represent the average of all replicate treatments; lower saturation points

1280 represent individual treatment measurements. Horizontal dashed lines denote 2 standard

1281 deviations of the phenotypes from all neutral gene controls. Up to 15 genes with the strongest

1282 phenotypes greater than 2 standard deviations (neutral gene controls) in either direction are

1283 listed.

1284

1285 
bioRxiv preprint doi: https://doi.org/10.1101/2020.12.22.423985; this version posted December 22, 2020. The copyright holder for this preprint (which was not certified by peer review) is the author/funder, who has granted bioRxiv a license to display the preprint in perpetuity. It is made available under aCC-BY 4.0 International license.

Figure 3 - Figure Supplement 6

a WT insertion

deletion $<50 \mathrm{nt}$ Donor $\mathrm{j}$

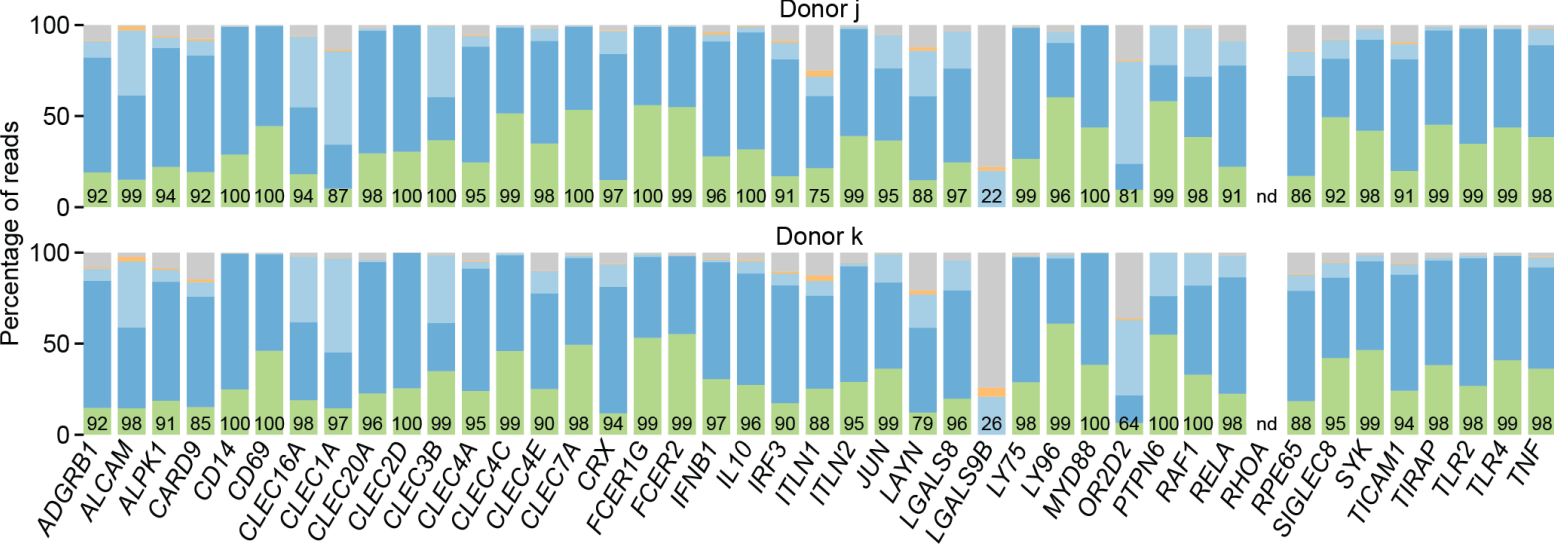

b
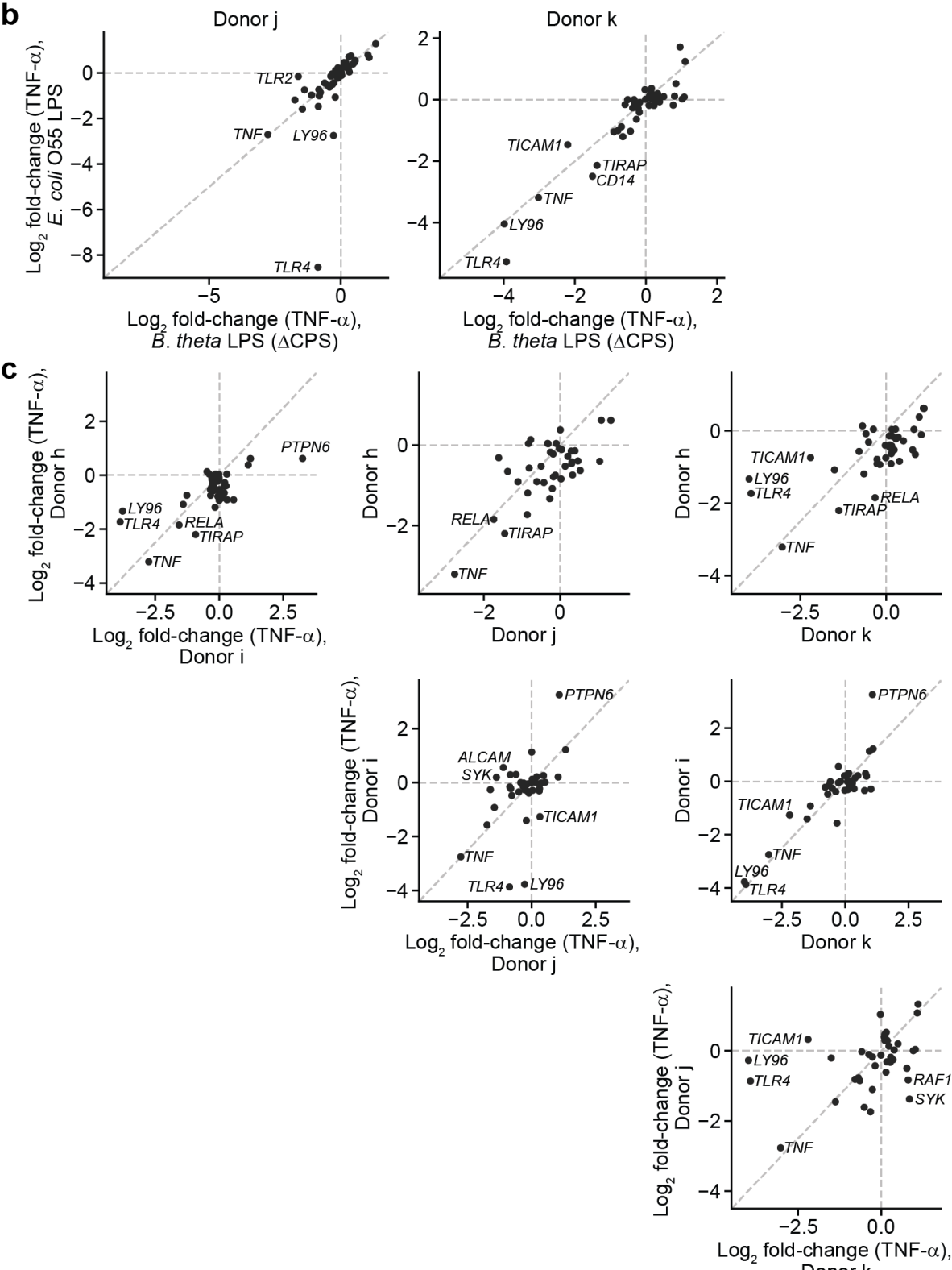
1287 Figure 3 - Figure Supplement 6. Validation of screen results in moDCs from two additional,

1288 independent donors. (a) Percentage of genomic DNA reads assigned to different classes of

1289 outcomes after targeting indicated loci in cells from two independent donors. Labeled values

1290 indicate total percentage of reads with a non-WT sequence. Editing efficiency was not quantified

1291 for RHOA. (b) $\log _{2}$ fold-change in TNF- $\alpha$ production by knockout moDCs challenged with 10

$1292 \mathrm{ng} / \mathrm{mL}$ E. coli O55 LPS or $100 \mathrm{ng} / \mathrm{mL}$ B. theta LPS relative to production in moDCs with

1293 knockout of neutral genes, normalized to cell counts. For donor j, knockout of TLR2 decreased

1294 the response to $B$. theta LPS but not E. coli LPS. Other genes with large $\log _{2}$ fold-changes are

1295 also labeled. Each data point represents data from two treatment replicates and a single cell count

1296 measurement. (c) $\log _{2}$ fold-change in TNF- $\alpha$ production by knockout moDCs challenged with

$1297100 \mathrm{ng} / \mathrm{mL}$ B. theta LPS from 4 independent donors, relative to production in moDCs with

1298 knockout of neutral genes. Data for donors $\mathrm{h}$ and $\mathrm{i}$ are derived from the arrayed screen. Genes

1299 with $\mid \log _{2}$ fold-change $\mid>1.8$ or $\Delta \log _{2}$ fold change $>1.3$ are labeled. 


\section{Supplemental File Legends}

1303 Supplementary File 1. Sequences of sgRNAs and amplicon PCR primers used in this work,

1304 including details on sgRNA binding sites.

1305 Supplementary File 2. Counts corresponding to different outcomes, corrected for amplicon size,

1306 after amplicon sequencing for all donors except donors $\mathrm{h}$ and $\mathrm{i}$.

1307 Supplementary File 3. Raw and processed TNF- $\alpha$ and IL-10 ELISA and cell count data for the 1308 genetic screens.

1309 Supplementary File 4. Counts corresponding to different outcomes, corrected for amplicon size, 1310 after amplicon sequencing for donors $\mathrm{h}$ and $\mathrm{i}$. 